A variational approach to coarse-graining of equilibrium and non-equilibrium atomistic description at finite temperature

Y. Kulkarni, J. Knap, M. Ortiz

April 30, 2007

Journal of the Mechanics and physics of Solids 
This document was prepared as an account of work sponsored by an agency of the United States Government. Neither the United States Government nor the University of California nor any of their employees, makes any warranty, express or implied, or assumes any legal liability or responsibility for the accuracy, completeness, or usefulness of any information, apparatus, product, or process disclosed, or represents that its use would not infringe privately owned rights. Reference herein to any specific commercial product, process, or service by trade name, trademark, manufacturer, or otherwise, does not necessarily constitute or imply its endorsement, recommendation, or favoring by the United States Government or the University of California. The views and opinions of authors expressed herein do not necessarily state or reflect those of the United States Government or the University of California, and shall not be used for advertising or product endorsement purposes. 


\title{
A variational approach to coarse-graining of equilibrium and non-equilibrium atomistic description at finite temperature
}

\author{
Yashashree Kulkarni, Jaroslaw Knap and Michael Ortiz \\ Graduate Aeronautical Laboratories \\ California Institute of Technology \\ Pasadena, CA 91125, USA
}

April 22, 2007

\begin{abstract}
The aim of this paper is the development of equilibrium and non-equilibrium extensions of the quasicontinuum (QC) method. We first use variational mean-field theory and the maximum-entropy formalism for deriving approximate probability distribution and partition functions for the system. The resulting probability distribution depends locally on atomic temperatures defined for every atom and the corresponding thermodynamic potentials are explicit and local in nature. The method requires an interatomic potential as the sole empirical input. Numerical validation is performed by simulating thermal equilibrium properties of selected materials using the LennardJones pair potential and the EAM potential and comparing with molecular dynamics results as well as experimental data. The max-ent variational approach is then taken as a basis for developing a three-dimensional non-equilibrium finite temperature extension of the quasicontinuum method. This extension is accomplished by coupling the local temperature-dependent free energy furnished by the max-ent approximation scheme to the heat equation in a joint thermo-mechanical variational setting. Results for finite-temperature nanoindentation tests demonstrate the ability of the method to capture non-equilibrium transport properties and differentiate between slow and fast indentation.
\end{abstract}

\section{Introduction}

The aim of this paper is the development of equilibrium and non-equilibrium extensions of the quasicontinuum (QC) method of Tadmor et al. [27] to finite temperature. A number of finite-temperature extensions of QC have been proposed in the past within the framework of equilibrium statistical mechanics and thermodynamics (cf, e. g., [7, 22]). Whereas these 
extensions are powerful and effective for application to equilibrium problems, e. g., systems at uniform temperature, they cannot be applied to systems away from equilibrium such as involving heat conduction, mass transport, and other non-equilibrium phenomena.

The path that we follow for deriving a non-equilibrium QC extension is as follows. Instead of resorting to equilibrium statistical mechanics, e. g., in the form of Gibbs canonical ensemble, as in previous finite-temperature extensions of $\mathrm{QC}$, we directly approximate the probability density function of finding the system in a certain state, not necessarily an equilibrium state. We do this by recourse to variational mean-field theory and the maximumentropy (max-ent) formalism for deriving approximate probability distribution and partition functions for the system. We envision systems of atoms whose state is defined by the position and linear momentum of each atom and that undergo Lagrangian dynamics defined by an empirical interatomic potential. In formulating the variational mean-field model we allow every atom to have its own local temperature and entropy, to be understood here as parameters that determine the local statistics of the atom. The max-ent variational principle then supplies the most likely probability density function within the assumed mean-field class and consistent with all constraints on the system (e. g., [28]) and, as a byproduct, the dependence of the local atomic entropy on the local atomic temperature.

For simplicity, we restrict attention to macroscopic processes that are quasi-static. Under these conditions, the net result of the max-ent procedure is to define a non-equilibrium free energy depending on the positions and temperatures of all atoms. Conveniently, for several interatomic potentials of interest, including Lennard-Jones (LJ) and Embedded-Atom Method (EAM), the non-equilibrium free energy can be computed explicitly up to numerical quadratures, and the result may be regarded as a temperature-dependent interatomic potential. This structure greatly facilitates implementation, which is reduced to replacing ordinary interatomic potentials by temperature-dependent ones.

For a given temperature field, the stable equilibrium configurations of the system follow from free-energy minimization. The chief remaining step in the development of the method therefore concerns the computation of the evolving temperature field. We accomplish this by coupling the free-energy minimization problem to a diffusion form of the energy balance equation. The proper form of the coupling is suggested by the variational formulation of coupled thermo-mechanical problems of Yang et al. [30]. This framework ensures that the coupled problem has a joint variational structure and, in particular, the time-discretized problem entails the minimization of a joint potential of displacement and temperature. In addition, the variational framework uniquely determines the precise manner in which mechanical work is converted into heat, a feature of the behavior of the system that must be modeled in other approaches. The coupling of the free energy to the energy equation introduces an empirical constant, namely, the heat conduction tensor, in addition to the empirical interatomic potential. In particular, the heat conductivity in the model does not follow as a result of the QC coarse-graining or any other form of upscaling of the molecular dynamics. However, in metals the electronic density contributes significantly to the heat conductivity and interatomic empirical potentials cannot possibly predict that property accurately. Therefore, accounting for heat conduction empirically is justified, albeit somewhat 
expedient, in those materials.

It bears emphasis that at no time in this procedure equilibrium statistical mechanics is invoked to define temperature and entropy or to determine the probability density function of the system. In particular, as used here, the terms temperature, entropy and free energy have non-standard meanings. Thus, unlike the conventional temperature and entropy defined in equilibrium thermodynamics and statistical mechanics, the local temperatures and entropies that arise in the present theory are parameters that the mean-field probability density function is endowed with. For non-interacting atoms, the local temperatures and entropies do indeed coincide with the equilibrium values of each one of the atoms regarded as an isolated system in thermodynamic equilibrium, which justifies the use of terminology. Likewise, the non-equilibrium free energy that arises in the present theory is defined formally from the mean-field probability density function and reduces to the equilibrium free energy of the system when the temperature field is uniform.

The plan of the paper is as follows: Section 2 provides a concise review of the fundamental postulates of statistical mechanics and the general framework for variational mean field theories. In section 3, this variational formulation is used to obtain local max-ent probability distribution functions for an atomistic system. The derivation of effective thermodynamic potentials is presented in section 4. In section 5, we show some example calculations for the Lennard-Jones pair potential and the EAM many-body interaction potential. Section 6 discusses the results for some numerical validation tests. Specifically, we calculate the specific heat and the thermal expansion coefficient of argon and copper using the LennardJones potential, and the embedded-atom method potential, respectively and compare with experimental data and molecular dynamics results. Next, we briefly review the static theory of the quasicontinuum in section 7 . Section 8 extends this framework to equilibrium thermodynamic processes. A convergence analysis of the method for homogeneous deformations such as uniform thermal expansion is also presented. In section 9, we review the variational formulation for coupled thermo-mechanical problems. Section 10 presents the details of the non-equilibrium finite temperature formulation of the quasicontinuum method. Finally, in section 11, results for some nanoindentation tests are discussed that demonstrate the ability of the method to capture non-equilibrium transport properties and simulate slow and fast microstructural evolution.

\section{General framework}

Let us consider a system of $\mathrm{N}$ atoms in configuration space $X$. Let $\boldsymbol{q} \in X \equiv \mathbb{R}^{3 N}$ represent the array of atomic positions and $\boldsymbol{p} \in Y \equiv \mathbb{R}^{3 N}$ be the array of corresponding momenta. Let $\boldsymbol{q}_{a} \in \mathbb{R}^{3}$ and $\boldsymbol{p}_{a} \in \mathbb{R}^{3}$ denote the position and the momentum of atom $a$. For simplicity of subsequent calculations, let $\boldsymbol{q}$ and $\boldsymbol{p}$ be defined as mass-reduced coordinates. Then, the Hamiltonian of the system is

$$
H(\boldsymbol{q}, \boldsymbol{p})=\frac{1}{2}|\boldsymbol{p}|^{2}+V(\boldsymbol{q})
$$


where $V(\boldsymbol{q})$ is the potential energy of the system expressed through the use of appropriate interatomic potentials. Thus, $(\boldsymbol{q}, \boldsymbol{p})$ denotes a point in the phase space $X \times Y$. Any function $f(\boldsymbol{q}, \boldsymbol{p})$ whose instantaneous value can be completely determined by the microstate, i.e., the instantaneous positions and momenta of the atoms, is referred to as a phase function. According to a fundamental premise of statistical mechanics [3, 17, 28], there exists a function, $p(\boldsymbol{q}, \boldsymbol{p}) \geq 0$, known as the probability distribution function and interpreted as the probability that the system be at point $(\boldsymbol{q}, \boldsymbol{p})$ in the phase space. Then, when the system attains equilibrium, any macroscopic quantity can be computed as a phase average of an appropriate phase function, $f(\boldsymbol{q}, \boldsymbol{p})$, where the phase average is defined with respect to $p(\boldsymbol{q}, \boldsymbol{p})$ as

$$
\langle f\rangle \equiv \frac{1}{N ! h^{3 N}} \int_{\Gamma} p f d \boldsymbol{q} d \boldsymbol{p}
$$

where we have used the following to simplify the notation:

$$
d \boldsymbol{q} d \boldsymbol{p} \equiv \prod_{a=1}^{N} \prod_{i=1}^{3} d q_{a i} d p_{a i} .
$$

$\Gamma$ denotes the phase space and $h$ is the Planck's constant. The factor $\left(N ! h^{3 N}\right)^{-1}$ arises from taking the classical limit of the analog of the phase averaging operation in quantum mechanics. It is also essential for the entropy to be extensive in classical statistical thermodynamics $[3,17]$.

In order to develop an averaging scheme for the atomistic system at finite temperature, the first step is to construct an appropriate probability distribution function which we accomplish by way of a mean field approximation as described in the next section. Mean field theory was developed essentially as an approximation tool for facilitating a theoretical treatment of critical phenomena such as phase transitions [2, 3, 17]. The basic idea is to study one particle in the system and treat its interactions with the neighboring particles as an average molecular field exerted by the atom's environment. This significantly reduces the degrees of freedom in the problem. The variational framework for deriving generalized mean field theories is implemented as follows: First a simplified functional form with free unspecified parameters is chosen as the trial probability distribution function. We denote it by $p$. We also let the approximate free energy obtained using this trial function be $F_{p}$. Since the effect of the ambience of a particle is approximated as a "mean field", the particles are treated as being statistically independent and hence the trial probability distribution is obtained as a product of local probability distribution functions

$$
p=\prod_{a=1}^{N} p_{a} .
$$

As will be described in the next section, the trial functional form for $p(\boldsymbol{q}, \boldsymbol{p})$ may be derived using the principle of maximum entropy. Then, the trial probability distribution function that best approximates the actual probability distribution function is determined by minimizing $F_{p}$ with respect to the unspecified parameters in $p$. This claim is based on a result 
known as the Bogoliubov's inequality, which states that the approximate free energy based on any probability distribution provides an upper bound for the exact free energy of the system [2]. That is,

$$
F \leq F_{p}
$$

for any $p$ satisfying the basic properties of a probability distribution. The exact free energy of the system, $F$, is defined by the relation [17]

$$
e^{-\beta F}=\left\langle e^{-\beta H}\right\rangle \text {, }
$$

where

$$
\beta=\frac{1}{k_{B} T},
$$

while $F_{p}$ is obtained as a Legendre transformation of the internal energy as

$$
\begin{aligned}
F_{p} & =E_{p}-T S_{p} \\
& =\langle H\rangle_{p}+k_{B} T\langle\log p\rangle_{p}
\end{aligned}
$$

where we have made use of the statistical definitions of the internal energy and entropy of the system. The temperature and entropy are related by the equilibrium relation

$$
T=\left(\frac{\partial E}{\partial S}\right)_{\boldsymbol{q}}
$$

For details of the proof of the inequality, we refer the readers to [2].

\section{Local max-ent distribution}

We now wish to determine a trial probability distribution function for the system under consideration based on the principle of maximum entropy [10, 12, 31, 5]. To this end, we define the global entropy of the system as postulated by Boltzmann

$$
S=-\frac{k_{B}}{N ! h^{3 N}} \int_{\Gamma} p \log p d \boldsymbol{q} d \boldsymbol{p}
$$

where $k_{B}$ is the Boltzmann constant introduced as a proportionality constant. The principle of maximum entropy is very well established in the field of statistical mechanics and has its origin in the information-theoretical point of view of the notion of entropy. Information theory was first introduced in statistical mechanics by Jaynes [10]. From this perspective, entropy is defined as a measure of the uncertainty in the information about a system of particles. For instance, let us consider a thought experiment of observing a given system at an arbitrary instant of time. Any point $(\boldsymbol{q}, \boldsymbol{p})$ in the phase space accessible to the system constitutes an outcome. Then, the entropy defined by eq. (3.1) is the uncertainty associated with the experiment. The principle of maximum entropy then states that the least biased 
probability distribution function maximizes the entropy of the system subject to all the imposed constraints or the information about the system which is already known.

Thus, our objective is to find the probability distribution that maximizes the entropy of the system subject to certain constraints. First, the probability distribution should satisfy the normalization condition

$$
\frac{1}{N ! h^{3 N}} \int_{\Gamma} p d \boldsymbol{q} d \boldsymbol{p}=1
$$

Suppose in addition that we have additional knowledge of the configuration of the ensemble. In particular, suppose that we know that atom $a$ moves in the vicinity of point $\overline{\boldsymbol{q}}_{a}$ with standard deviation $\sqrt{3} \tau_{a}$ and has momentum in the vicinity of $\overline{\boldsymbol{p}}_{a}$ with standard deviation $\sqrt{3} \sigma_{a}$. Thus, $\overline{\boldsymbol{q}}_{a}$ and $\overline{\boldsymbol{p}}_{a}$ are the mean position and the mean momentum of atom $a$ defined as the first moment of $\boldsymbol{q}_{a}$ and $\boldsymbol{p}_{a}$, respectively,

$$
\left\langle\boldsymbol{q}_{a}\right\rangle=\overline{\boldsymbol{q}}_{a},\left\langle\boldsymbol{p}_{a}\right\rangle=\overline{\boldsymbol{p}}_{a}, \forall a=1, \ldots, N
$$

Physically, $\overline{\boldsymbol{q}}_{a}$ and $\overline{\boldsymbol{p}}_{a}$ may be interpreted as variables on the continuum scale following the dynamics of the system on the macroscopic time scale. For instance, for a quasistatic process, $\overline{\boldsymbol{p}}=0$ and $\overline{\boldsymbol{q}}$ represents an equilibrium configuration of the system. Taking second moments of $\boldsymbol{q}_{a}$ and $\boldsymbol{p}_{a}$ introduces the following constraints:

$$
\begin{aligned}
& \left\langle\left|\boldsymbol{q}_{a}-\overline{\boldsymbol{q}}_{a}\right|^{2}\right\rangle=3 \tau_{a}^{2}, \forall a=1, \ldots, N, \\
& \left\langle\left|\boldsymbol{p}_{a}-\overline{\boldsymbol{p}}_{a}\right|^{2}\right\rangle=3 \sigma_{a}^{2}, \forall a=1, \ldots, N .
\end{aligned}
$$

The factor of 3 is included merely to keep the subsequent expressions simple and to motivate the physical interpretation of these parameters. In order to simplify the interpretation further, we replace the parameter $\tau_{a}$ by $\omega_{a}$ defined as

$$
\omega_{a}=\frac{\sigma_{a}}{\tau_{a}}
$$

and having the unit of frequency. Since $\sigma_{a}$ and $\omega_{a}$ are defined as phase averages, they are also macroscopic variables. As we shall see in section 4.5, they establish a link between the energetics of the microscopic scale and the thermodynamic quantities. Using the expression for $\omega_{a}$ in eq. (3.4a) and adding the resulting equation to eq. (3.4b), we have

$$
\left\langle\left|\boldsymbol{p}_{a}-\overline{\boldsymbol{p}}_{a}\right|^{2}\right\rangle+\omega_{a}^{2}\left\langle\left|\boldsymbol{q}_{a}-\overline{\boldsymbol{q}}_{a}\right|^{2}\right\rangle=6 \sigma_{a}^{2}, \forall a=1, \ldots, N
$$

Introducing the $N+1$ constraints given by eq. (3.2) and eq. (3.6) as Lagrange multipliers [16], the extremum problem may be stated as

$$
\sup _{p}-\frac{k_{B}}{N ! h^{3 N}} \int_{\Gamma} p \log p+p \lambda+p \sum_{a=1}^{N} \beta_{a}\left[\left|\boldsymbol{p}_{a}-\overline{\boldsymbol{p}}_{a}\right|^{2}+\omega_{a}^{2}\left|\boldsymbol{q}_{a}-\overline{\boldsymbol{q}}_{a}\right|^{2}\right] d \boldsymbol{q} d \boldsymbol{p}
$$

where $\lambda$ corresponds to eq. (3.2) and the $N$ Lagrange multipliers $\beta_{a}$ correspond to eq. (3.6). Taking the variation of eq. (3.7) with respect to $p$ and enforcing stationarity yields the desired 
probability distribution function

$$
p(\boldsymbol{z} \mid \overline{\boldsymbol{z}},\{\sigma\},\{\omega\})=Z^{-1} \exp \left[-\sum_{a=1}^{N} \beta_{a}\left\{\left|\boldsymbol{p}_{a}-\overline{\boldsymbol{p}}_{a}\right|^{2}+\omega_{a}^{2}\left|\boldsymbol{q}_{a}-\overline{\boldsymbol{q}}_{a}\right|^{2}\right\}\right],
$$

where $Z=\exp [1+\lambda]$ is known as the partition function of the system. For economy of notation, we have also introduced $\boldsymbol{z} \equiv(\boldsymbol{q}, \boldsymbol{p})$ to denote the microstate and $\overline{\boldsymbol{z}},\{\sigma\}$ and $\{\omega\}$ to represent the corresponding arrays of macroscopic variables. Thus, $\overline{\boldsymbol{z}} \equiv(\overline{\boldsymbol{q}}, \overline{\boldsymbol{p}})$ and $\{\sigma\},\{\omega\} \in \mathbb{R}^{N}$. " " is used to separate the microscopic and the macroscopic variables. $Z$ may be evaluated analytically by substituting eq. (3.8) in eq. (3.2). The integration yields

$$
Z=\frac{1}{N ! h^{3 N}} \prod_{a=1}^{N}\left(\sqrt{\frac{\pi}{\beta_{a}}}\right)^{3}\left(\sqrt{\frac{\pi}{\beta_{a}}} \frac{1}{\omega_{a}}\right)^{3}
$$

By substituting eq. (3.8) in either eq. (3.6) or eq. (3.4b) and using eq. (3.9) we obtain the Lagrange multipliers as

$$
\beta_{a}=\frac{1}{2 \sigma_{a}^{2}}
$$

The final expressions for the max-ent probability distribution and partition function are

$$
\begin{aligned}
& p(\boldsymbol{z} \mid \overline{\boldsymbol{z}},\{\sigma\},\{\omega\})=Z^{-1} \exp \left[-\sum_{a=1}^{N} \frac{\left|\boldsymbol{p}_{a}-\overline{\boldsymbol{p}}_{a}\right|^{2}+\omega_{a}^{2}\left|\boldsymbol{q}_{a}-\overline{\boldsymbol{q}}_{a}\right|^{2}}{2 \sigma_{a}^{2}}\right] \\
& Z=\frac{1}{N ! h^{3 N}} \prod_{a=1}^{N}\left(\sqrt{2 \pi} \sigma_{a}\right)^{3}\left(\sqrt{2 \pi} \frac{\sigma_{a}}{\omega_{a}}\right)^{3} .
\end{aligned}
$$

It may be striking to note that $p$ and $Z$ do not depend on the interaction potential of the system. However, the dependence is implicit in the $\{\omega\}$ which are unspecified so far. We also observe that owing to the local constraints, the global partition function and probability distribution are derived naturally as products of terms associated with each atom. This enables us to write eq. (3.11a) and eq. (3.11b) as

$$
\begin{aligned}
& p(\boldsymbol{z} \mid \overline{\boldsymbol{z}},\{\sigma\},\{\omega\})=\prod_{a=1}^{N} p_{a}\left(\boldsymbol{z}_{a} \mid \overline{\boldsymbol{z}}_{a}, \sigma_{a}, \omega_{a}\right), \\
& Z=\prod_{a=1}^{N} Z_{a}
\end{aligned}
$$

We wish to emphasize that the local-equilibrium hypothesis $[12,31,5]$ is implicit in the statement of the constraints, (3.4a) and (3.4b), and forms a basis of all our later work. It postulates that if a system can be hypothetically split into subsystems, each very close to thermal equilibrium, then the thermodynamic relations hold within each cell. Thus, it 
assumes the existence of two relaxation times - the relaxation time for the establishment of statistical equilibrium in the whole system and another, much shorter, for establishing equilibrium within a small cell. This enables a rigorous definition of thermodynamic state variables such as temperature and entropy locally. Hence, the local-equilibrium hypothesis forms a fundamental premise of classical irreversible thermodynamics. Likewise, in our approach, it enables us to define phase averages locally and also introduce atomic notions of entropy and temperature.

The constraints also distinguish the max-ent distribution from the canonical distribution of Gibbs in that the latter is derived by imposing a constraint on the global energy of the system. For a system in thermal equilibrium, this may be stated as

$$
\langle H(\boldsymbol{q}, \boldsymbol{p})\rangle=E,
$$

where $H$ is the Hamiltonian, and $E$ the total internal energy of the system. The resulting probability distribution function has the form

$$
p(\boldsymbol{q}, \boldsymbol{p})=Z^{-1} \exp \left[-\frac{H}{k_{B} T}\right] .
$$

This form may also be used to derive local thermodynamic quantities by assuming local thermal equilibrium [12]. However, due to the difficulty of integrating this function in the case of anharmonic interaction potentials, the partition function and the thermodynamic potentials may be obtained analytically only for the harmonic approximation. In contrast, the probability distribution function given by (3.11a) involves Gaussian functions. Consequently, the phase averages may be computed analytically for many functions, or at least numerically by Gauss quadrature while retaining the anharmonic effect of the interatomic potentials in the macroscopic thermodynamic properties.

We also note that in this process of determining a simplified solution for the probability distribution by imposing additional constraints, we have introduced into the problem $2 \mathrm{~N}$ additional unknowns, $\{\sigma\}$ and $\{\omega\}$, referred to as the mean field parameters. Based on the variational theory of mean field approximation outlined earlier, these parameters are determined by minimizing the free energy of the system.

\section{Thermodynamic potentials}

Having constructed a suitable probability distribution, we can obtain the local forms of the desired thermodynamic potentials, namely, the entropy, the internal energy, and the free energy as described below. 


\subsection{Entropy}

Substituting eq. (3.11a) and eq. (3.11b) in the expression (3.1), the integral for the global entropy evaluates to

$$
S=k_{B}\left[-\log N !+3 N+3 \sum_{a=1}^{N} \log \frac{\sigma_{a}^{2}}{\hbar \omega_{a}}\right] .
$$

For a system with a very large number of particles, we use Sterling's formula [3]

$$
\log N ! \approx N \log N-N
$$

to reduce eq. (4.1) to

$$
S=k_{B}\left[-N \log N+4 N+3 \sum_{a=1}^{N} \log \frac{\sigma_{a}^{2}}{\hbar \omega_{a}}\right] .
$$

Since entropy is an extensive property of the system,

$$
S \equiv \sum_{a=1}^{N} S_{a}
$$

where

$$
S_{a}=3 k_{B} \log \frac{\sigma_{a}^{2}}{\hbar \omega_{a}}+4 k_{B}-k_{B} \log N
$$

can be interpreted as the contribution of atom $a$ to the total entropy. The relation (4.5) can be inverted to yield

$$
\sigma_{a}=\sqrt{\hbar \omega_{a}} \exp \left[\frac{S_{a}}{6 k_{B}}-\frac{4}{6}+\frac{1}{6} \log N\right] .
$$

This is an explicit expression for $\sigma_{a}$, and we use it henceforth to replace $\sigma_{a}$ by a function of $S_{a}$ and $\omega_{a}$.

\subsection{Internal energy}

In order to derive the internal energy of the system, we suppose that the atoms move according to a Hamiltonian $H(\boldsymbol{z})$. Then, the internal energy is defined as the phase average of the Hamiltonian of the system [28]. Suppose that the Hamiltonian has an additive structure

$$
H(\boldsymbol{z})=\sum_{a=1}^{N} H_{a}(\boldsymbol{z})
$$

and let $H_{a}(\boldsymbol{z})$ be of the form

$$
H_{a}(\boldsymbol{z})=\frac{1}{2}\left|\boldsymbol{p}_{a}\right|^{2}+V_{a}(\boldsymbol{q})
$$


Then, the expression for the internal energy becomes

$$
E(\overline{\boldsymbol{z}},\{S\},\{\omega\})=\frac{1}{N ! h^{3 N}} \sum_{a=1}^{N} \int_{\Gamma}\left[\frac{1}{2}\left|\boldsymbol{p}_{a}\right|^{2}+V_{a}(\boldsymbol{q})\right] p(\boldsymbol{z} \mid \overline{\boldsymbol{z}},\{S\},\{\omega\}) d \boldsymbol{q} d \boldsymbol{p} .
$$

The phase average of the kinetic energy can be computed analytically atom by atom and reduces to

$$
\left\langle\frac{1}{2}\left|\boldsymbol{p}_{a}\right|^{2}\right\rangle=\frac{1}{2}\left(3 \sigma_{a}^{2}+\left|\overline{\boldsymbol{p}}_{a}\right|^{2}\right)
$$

Unlike kinetic energy, the integration of $V_{a}(\boldsymbol{q})$ involves all the neighbors of atom $a$, and in most cases, cannot be computed analytically. Traditionally, the harmonic approximation is used in order to facilitate analytical calculations. However, in our approach, the phase integrals may be computed numerically even for anharmonic potentials by way of Gauss quadrature, and we defer the discussion on the numerical integration of the potential energy till section 5. Thus, for a system undergoing a quasistatic process, the internal energy has the form

$$
E(\overline{\boldsymbol{q}},\{S\},\{\omega\})=\frac{3}{2} \sum_{a=1}^{N} \hbar \omega_{a} \exp \left[\frac{S_{a}}{3 k_{B}}-\frac{4}{3}+\frac{1}{3} \log N\right]+\sum_{a=1}^{N}\left\langle V_{a}(\boldsymbol{q})\right\rangle,
$$

where we have made use of eq. (4.6) to replace $\sigma_{a}^{2}$.

\subsection{Equipartition of energy}

The equipartition of energy is a fundamental result of statistical mechanics which states that for a system in thermal equilibrium, each quadratic term in the Hamiltonian contributes $k_{B} T / 2$ to the mean Hamiltonian or the internal energy of the system, where the phase average is taken with respect to the canonical distribution [28]. In the max-ent method, we enforce the equipartition of energy through the local kinetic energy as

$$
\left\langle\frac{1}{2}\left|\boldsymbol{p}_{a}\right|^{2}\right\rangle=\frac{3}{2} k_{B} T_{a}
$$

Comparing this relation with eq. (4.10) for a quasi-static process yields a direct interpretation of $\sigma_{a}$ in terms of the local temperature:

$$
\sigma_{a}^{2}=k_{B} T_{a}
$$

Using this definition of $\sigma_{a}$ in eq. (4.5) gives an equilibrium relation between the local entropy and the local temperature:

$$
S_{a}=3 k_{B} \log \frac{k_{B} T_{a}}{\hbar \omega_{a}}+4 k_{B}-k_{B} \log N .
$$

As we shall see later, although the above expression is local, it is not independent of the atom's surrounding since $\omega_{a}$ contains the effect of the interactions of the atom with its neighbors. 


\subsection{Helmholtz free energy}

The Helmholtz free energy is defined as a Legendre transformation of the internal energy with respect to the entropy:

$$
F(\overline{\boldsymbol{q}},\{T\},\{\omega\})=\inf _{\{S\}}\left\{E(\overline{\boldsymbol{q}},\{S\},\{\omega\})-\sum_{a} T_{a} S_{a}\right\} .
$$

The minimization with respect to $S_{a}$ yields the equilibrium relation

$$
T_{a}=\frac{\partial E}{\partial S_{a}}(\overline{\boldsymbol{q}},\{S\},\{\omega\}) .
$$

Other thermodynamic potentials such as the Gibbs free energy and the enthalpy of the system may similarly be derived by appropriate Legendre transformations $[3,16]$. We also recall that the optimal value of the mean field parameters that we introduced can now be determined by minimizing the free energy. Thus, the complete problem of ascertaining the equilibrium configurations of a system undergoing a quasistatic process at finite temperature may be enunciated as

$$
\inf _{\overline{\boldsymbol{q}}} \inf _{\{\omega\}} F(\overline{\boldsymbol{q}},\{T\},\{\omega\}),
$$

where $F$ is the Helmholtz free energy of the system evaluated above.

As a concluding remark, we note that for a dynamic process, i.e., with $\overline{\boldsymbol{p}} \neq 0$, the minimization problem may be replaced by the canonical equations derived from the macroscopic Hamiltonian:

$$
\dot{\overline{\boldsymbol{q}}}=\frac{\partial \bar{H}}{\partial \overline{\boldsymbol{p}}} ; \quad \dot{\overline{\boldsymbol{p}}}=-\frac{\partial \bar{H}}{\partial \overline{\boldsymbol{q}}},
$$

where $\bar{H}=\langle H\rangle$. However, we shall not pursue this direction in our current work and shall restrict ourselves to quasistatic processes.

\subsection{Interpretation of the mean field parameters}

In this section we investigate the connection between our approach and statistical mechanics in order to obtain an interpretation of the mean field parameters. To this end, we consider a quasi-harmonic approximation for the potential energy of the system about an equilibrium configuration. By further assuming weak interactions between the atoms, the potential energy may be reduced as

$$
V(\boldsymbol{q}) \approx V(\overline{\boldsymbol{q}})+\sum_{a} \frac{1}{2} \boldsymbol{x}_{a}^{\mathrm{T}} \mathrm{K}_{a}(\overline{\boldsymbol{q}}) \boldsymbol{x}_{a}=\sum_{a} V_{a}(\boldsymbol{q}),
$$

where $\mathrm{K}_{a}$ is the $3 \times 3$ local dynamical matrix associated with each atom and defined as

$$
\mathrm{K}_{a}(\overline{\boldsymbol{q}})=\left.\frac{\partial^{2} V}{\partial \boldsymbol{q}_{a}^{2}}\right|_{\boldsymbol{q}_{a}=\overline{\boldsymbol{q}}_{a}} .
$$


and

$$
\boldsymbol{x}_{a}=\boldsymbol{q}_{a}-\overline{\boldsymbol{q}}_{a} .
$$

This is known as the local quasi-harmonic approximation. It assumes the system to be in weak interaction so that each atom is regarded as a harmonic oscillator with its neighbors fixed at their current mean positions. This approximation is found to be adequate for temperatures up to half the melting point of the material [18]. We note that the coupling of an atom with its neighborhood is retained through the dependence of the local dynamical matrix on the macroscopic variables $\overline{\boldsymbol{q}}$. Then, the phase average of the potential energy may be computed analytically as

$$
\left\langle V_{a}\right\rangle=V_{a}(\overline{\boldsymbol{q}})+\frac{1}{2} \frac{\sigma_{a}^{2}}{\omega_{a}^{2}} \operatorname{Tr} \mathrm{K}_{a}(\overline{\boldsymbol{q}}) .
$$

where $\operatorname{Tr}$ denotes the trace of the matrix. On substituting the above in eq. (4.11) the internal energy of the system becomes

$$
E(\overline{\boldsymbol{q}},\{S\},\{\omega\})=\sum_{a} V_{a}(\overline{\boldsymbol{q}})+\sum_{a} \frac{1}{2}\left[3+\frac{1}{\omega_{a}^{2}} \operatorname{Tr} \mathrm{K}_{a}(\overline{\boldsymbol{q}})\right] \frac{h \omega_{a}}{2 \pi} \exp \left[\frac{S_{a}}{3 k_{B}}-\frac{4}{3}+\frac{1}{3} \log N\right],
$$

The equilibrium relation (4.16) yields

$$
T_{a}=\frac{1}{6 k_{B}}\left[3+\frac{1}{\omega_{a}^{2}} \operatorname{Tr} \mathbf{K}_{a}(\overline{\boldsymbol{q}})\right] \frac{h \omega_{a}}{2 \pi} \exp \left[\frac{S_{a}}{3 k_{B}}-\frac{4}{3}+\frac{1}{3} \log N\right] .
$$

We invert the above relation to obtain $S_{a}$ in terms of $T_{a}$. Substituting it in eq. (4.22) gives the internal energy as a function of the local temperatures:

$$
E(\overline{\boldsymbol{q}},\{T\},\{\omega\})=V(\overline{\boldsymbol{q}})+\sum_{a} 3 k_{B} T_{a},
$$

which is a well known result from statistical mechanics in agreement with the equipartition of energy. By substituting these expressions for the internal energy and the entropy as functions of the temperature in eq. (4.15), the free energy becomes

$F(\overline{\boldsymbol{q}},\{T\},\{\omega\})=V(\overline{\boldsymbol{q}})-3 k_{B} \sum_{a} T_{a}\left[\log \frac{6 k_{B} T_{a}}{\hbar}-\log \left\{\left[3+\frac{1}{\omega_{a}^{2}} \operatorname{TrK}_{a}(\overline{\boldsymbol{q}})\right] \omega_{a}\right\}+\frac{4}{3}-\frac{1}{3} \log N\right]$.

Minimizing $F$ with respect to $\omega_{a}$ gives

$$
\omega_{a}^{2}=\frac{1}{3} \operatorname{Tr} K_{a}=\frac{1}{3} \sum_{i=1}^{3} \bar{\omega}_{i a}^{2}(\overline{\boldsymbol{q}})
$$

where $\bar{\omega}_{i a}$ denotes the three frequencies associated with the atom $a$. This implies that for a quasi-harmonic approximation, $\omega_{a}^{2}$ equals the arithmetic mean of the squares of the quasiharmonic frequencies associated with that atom. Furthermore, substituting this in eq. (4.23) verifies that at equilibrium

$$
\sigma_{a}^{2}=k_{B} T_{a},
$$


as derived in section 4 . Thus, for a system with a general anharmonic potential energy, $\omega_{a}$ provides an approximate average of the local frequencies of the atom $a$. This is an important result since it reveals the physical nature of the parameters $\omega_{a}$ and confirms that the arrays $\{\omega\}$ and $\{\sigma\}$ establish a link between the energetics of the microscopic dynamics and the effective macroscopic energy of the system.

As a closing remark, we would like to mention that for a perfect, infinite, isotropic crystal with a local quasi-harmonic approximation which is subjected to uniform temperature, the max-ent probability distribution and the thermodynamic potentials are identical to those furnished by the Gibbs canonical distribution [15].

\section{Phase averages by Gauss quadrature}

Sections 3 and 4 describe the procedure for arriving at temperature-dependent effective potentials based on the local max-ent approximation scheme. Recalling eq. (4.11), we now wish to evaluate the phase average of the potential energy $V(\boldsymbol{q})$. To this end, we suppose that each function $V_{a}(\boldsymbol{q})$ involves a small number of neighboring atoms. Then, the integrals in $\left\langle V_{a}(\boldsymbol{q})\right\rangle$ are likewise of small dimensionality and can effectively be computed by means of Gaussian quadrature, i.e., with integration points and weights corresponding to a Gaussian weight function. This is due to the specific form of the max-ent probability densities. We begin by considering an $n$-body interaction potential, $\phi\left(\boldsymbol{q}_{1}, \ldots, \boldsymbol{q}_{n}\right)$. The expectation value of this function is computed as

$$
\begin{aligned}
\left\langle\phi\left(\boldsymbol{q}_{1}, \ldots, \boldsymbol{q}_{n}\right)\right\rangle & =\frac{1}{N ! h^{3 N}} \int_{\Gamma} \phi\left(\boldsymbol{q}_{1}, \ldots, \boldsymbol{q}_{n}\right) \prod_{a=1}^{n} p_{a} d \boldsymbol{p}_{a} d \boldsymbol{q}_{a} \\
& =\left(\frac{1}{\sqrt{\pi}}\right)^{3 n} \int_{-\infty}^{\infty} \cdots \int_{-\infty}^{\infty} \tilde{\phi}\left(\boldsymbol{x}_{1}, \ldots, \boldsymbol{x}_{n}\right) \exp \left(-\left|\boldsymbol{x}_{1}\right|^{2}-\cdots-\left|\boldsymbol{x}_{n}\right|^{2}\right) d \boldsymbol{x}_{1} \cdots d \boldsymbol{x}_{n}
\end{aligned}
$$

where

$$
\tilde{\phi}\left(\boldsymbol{x}_{1}, \ldots, \boldsymbol{x}_{n}\right)=\phi\left(\boldsymbol{q}_{1}\left(\boldsymbol{x}_{1}\right), \ldots, \boldsymbol{q}_{n}\left(\boldsymbol{x}_{n}\right)\right) .
$$

Eq. (5.1b) is the result of a change of variables:

$$
\boldsymbol{x}_{a}=\frac{1}{\sqrt{2}} \frac{\omega_{a}}{\sigma_{a}}\left(\boldsymbol{q}_{a}-\overline{\boldsymbol{q}}_{a}\right) .
$$

The multiple integral in eq. (5.1b) is of dimension $3 n$ and may be computed by using the Hermite-Gauss quadrature rule appropriate for the dimension of the space $[25,8]$. An $M$ point quadrature reduces the integral to

$$
\left\langle\phi\left(\boldsymbol{q}_{1}, \ldots, \boldsymbol{q}_{n}\right)\right\rangle \approx\left(\frac{1}{\sqrt{\pi}}\right)^{3 n} \sum_{k=1}^{M} \tilde{\phi}\left(\xi_{k}\right) W_{k},
$$


where $k$ denotes a quadrature point in phase space, $W_{k}$ is the corresponding weight and $\xi$ is a vector of dimension $3 n$ :

$$
\xi=\left(\boldsymbol{x}_{1}, \ldots, \boldsymbol{x}_{n}\right) \in \mathbb{R}^{3 n} .
$$

In our calculations, we use quadrature rules for multiple integrals developed by Stroud $[25,24]$. A limiting factor in the choice of quadrature formulae is the dimension of the domain of integration which is the configurational phase space of the interacting atoms. Thus, for a pair potential, we may use $3^{\text {rd }}$ and $5^{\text {th }}$ degree quadrature rules whereas for manybody potentials, we are restricted to $3^{\text {rd }}$ degree quadrature. An overview of the quadrature rules is presented in Appendix A.

Thus, the max-ent distribution provides a way to compute an approximate internal energy of the system, which should be exact for up to $3^{\text {rd }}$ or $5^{\text {th }}$ order Taylor expansion of the potential energy about an equilibrium configuration. An important implication of this higher order approximation is the ability to account for the anharmonicity of the interaction potential in studying the thermodynamic behavior of materials.

We now demonstrate the above calculations for the Lennard-Jones pair potential and the embedded atom method involving many-body interactions. Since our goal is the minimization of free energy, we also provide the expressions for the derivatives of the energy with respect to the atomic positions and the mean field variables, $\{\omega\}$. We also remark that these are mere examples to illustrate the generality of applying the max-ent distribution to any empirical interatomic potential and crystal structure.

\subsection{Lennard-Jones potential}

The phase average of the potential energy based on the Lennard-Jones pair potential is of the form

$$
\langle V\rangle=\frac{1}{2} \sum_{a} \sum_{b}\left\langle\phi\left(r_{a b}\right)\right\rangle
$$

where

$$
\phi(r)=4 \epsilon\left[\left(\frac{\sigma}{r}\right)^{12}-2\left(\frac{\sigma}{r}\right)^{6}\right]
$$

represents the bond energy and $r_{a b}$ denotes the distance between atoms $a$ and $b$. Typically, $b$ denotes the nearest neighbors of atom $a$. Since the potential involves only pairwise interactions, the phase averages can be computed over individual bonds involving two atoms. Consequently, the dimension of the domain of integration is 6. Applying the change of variables given in eq. (5.2), we have

$$
\begin{aligned}
r_{a b} & =\left|\boldsymbol{q}_{a}-\boldsymbol{q}_{b}\right| \\
& =\left|\sqrt{2} \frac{\sigma_{a}}{\omega_{a}} \boldsymbol{x}_{a}-\sqrt{2} \frac{\sigma_{b}}{\omega_{b}} \boldsymbol{x}_{b}+\overline{\boldsymbol{q}}_{a}-\overline{\boldsymbol{q}}_{b}\right| .
\end{aligned}
$$

Then, the energy of each bond calculated by quadrature is

$$
\left\langle\phi\left(r_{a b}\right)\right\rangle \approx\left(\frac{1}{\sqrt{\pi}}\right)^{6} \sum_{k=1}^{M} \phi\left(\tilde{\xi}_{k}\right) W_{k} .
$$


Taking the derivative of the energy with respect to the atomic positions yields

$$
\begin{aligned}
\frac{\partial}{\partial \overline{\boldsymbol{q}}_{a}}\langle V\rangle & =\sum_{b} \frac{\partial}{\partial \overline{\boldsymbol{q}}_{a}}\left\langle\phi\left(r_{a b}\right)\right\rangle \\
& =\left(\frac{1}{\sqrt{\pi}}\right)^{6} \sum_{b} \sum_{k=1}^{M} \phi^{\prime}\left(r_{a b}\left(\xi_{k}\right)\right) \frac{\boldsymbol{r}_{a b}}{r_{a b}} W_{k},
\end{aligned}
$$

where prime denotes differentiation with respect to $r$. Taking the derivative of the internal energy with respect to $\omega_{a}$ yields

$$
\begin{aligned}
\frac{\partial}{\partial \omega_{a}}\langle V\rangle & =\sum_{b} \frac{\partial}{\partial \omega_{a}}\left\langle\phi\left(r_{a b}\right)\right\rangle \\
& =-\frac{1}{\sqrt{2}}\left(\frac{1}{\sqrt{\pi}}\right)^{6} \sum_{b} \sum_{k=1}^{M} \frac{\sigma_{a}}{\omega_{a}^{2}} \frac{\phi^{\prime}\left(r_{a b}\left(\xi_{k}\right)\right)}{r_{a b}}\left[\boldsymbol{r}_{a b} \cdot \boldsymbol{x}_{a}^{k}\right] W_{k}
\end{aligned}
$$

and

$$
\frac{\partial}{\partial \omega_{a}}\left\langle\frac{1}{2}\left|\boldsymbol{p}_{a}\right|^{2}\right\rangle=\frac{3}{2} \hbar \exp \left[\frac{S_{a}}{3 k_{B}}-\frac{4}{3}+\frac{1}{3} \log N\right]
$$

where eq. (5.11) gives the contribution of the kinetic energy.

\subsection{EAM potential}

The potential energy based on the embedded-atom method $[4,11]$ is of the form

$$
V=\sum_{a} F\left(\rho_{a}\right)+\frac{1}{2} \sum_{a} \sum_{b} \phi\left(r_{a b}\right)
$$

In addition to a pair potential, we now have a term $F\left(\rho_{a}\right)$ for each atom, which is known as the embedding function and which depends on the electron density at site $a$ due to all its neighbors. Here, we present the expressions only for the embedding term. Let us define

$$
V_{2}=\sum_{a=1}^{N} F\left(\rho_{a}\right)
$$

Then,

$$
\left\langle V_{2}\right\rangle=\sum_{a=1}^{N}\left\langle F\left(\rho_{a}\right)\right\rangle=\sum_{a=1}^{N}\left(\frac{1}{\sqrt{\pi}}\right)^{3 n} \sum_{k=1}^{M} F\left(\rho_{a}\left(\xi_{k}\right)\right) W_{k},
$$

where $n$ includes the atom $a$ and its contributing neighbors. For an fcc crystal with nearestneighbor interactions, $n=13$ for an atom having all its neighbors. The derivative of $\left\langle V_{2}\right\rangle$ 
with respect to the atomic positions is of the form

$$
\begin{aligned}
\frac{\partial}{\partial \overline{\boldsymbol{q}}_{a}}\left\langle V_{2}\right\rangle & =\left(\frac{1}{\sqrt{\pi}}\right)^{3 n_{a}} \sum_{b} \sum_{k=1}^{M_{a}} F^{\prime}\left(\rho_{a}\left(\xi_{k}\right)\right) f^{\prime}\left(r_{a b}\left(\xi_{k}\right)\right) \frac{\boldsymbol{r}_{a b}}{r_{a b}} W_{k} \\
& +\sum_{b}\left(\frac{1}{\sqrt{\pi}}\right)^{3 n_{b}} \sum_{l=1}^{M_{b}} F^{\prime}\left(\rho_{b}\left(\xi_{l}\right)\right) f^{\prime}\left(r_{a b}\left(\xi_{l}\right)\right) \frac{\boldsymbol{r}_{a b}}{r_{a b}} W_{l}
\end{aligned}
$$

where

$$
F^{\prime}=\frac{d F}{d \rho}, f^{\prime}=\frac{d f}{d r} .
$$

Similarly, the derivative of $\left\langle V_{2}\right\rangle$ with respect to the local mean field parameters is

$$
\begin{aligned}
\frac{\partial}{\partial \omega_{a}}\left\langle V_{2}\right\rangle & =-\frac{1}{\sqrt{2}}\left(\frac{1}{\sqrt{\pi}}\right)^{3 n_{a}} \frac{\sigma_{a}}{\omega_{a}^{2}} \sum_{b} \sum_{k=1}^{M_{a}} F^{\prime}\left(\rho_{a}\left(\xi_{k}\right)\right) f^{\prime}\left(r_{a b}\left(\xi_{k}\right)\right)\left[\boldsymbol{r}_{a b} \cdot \boldsymbol{x}_{a}^{k}\right] W_{k} \\
& -\frac{1}{\sqrt{2}} \sum_{b}\left(\frac{1}{\sqrt{\pi}}\right)^{3 n_{b}} \frac{\sigma_{a}}{\omega_{a}^{2}} \sum_{l=1}^{M_{b}} F^{\prime}\left(\rho_{b}\left(\xi_{l}\right)\right) f^{\prime}\left(r_{a b}\left(\xi_{l}\right)\right)\left[\boldsymbol{r}_{a b} \cdot \boldsymbol{x}_{a}^{l}\right] W_{l} .
\end{aligned}
$$

In eq. (5.15) and eq. (5.16), $n_{a}$ is the total number of atoms in the neighborhood of atom $a$, and $M_{a}$ is the number of quadrature points used to compute the phase average $\left\langle F\left(\rho_{a}\right)\right\rangle$. Using the $3^{\text {rd }}$ degree quadrature formula for an atom in an fcc crystal with all its nearest neighbors present requires 78 quadrature points.

It bears emphasis that although $\omega$ is introduced as a local parameter associated with each atom, eq. (5.10b) and eq. (5.16) show that the minimization of the free energy with respect to $\omega_{a}$ cannot be achieved atom by atom. This reaffirms the non-local nature of $\{\omega\}$, indicating that it is related to the atomic interactions.

\section{Numerical validation}

For the purpose of numerical validation, we present our calculations of the specific heat and the coefficient of thermal expansion for solid argon and copper. Solid argon is modelled using the Lennard-Jones pair potential whereas two different potentials are used for copper, namely, the EAM potential proposed by Johnson [11] and another proposed by Sutton and Chen [26], both of which are based on the embedded-atom method [4].

The sample used for these tests is a cube of an fcc crystal consisting of 108 atoms with periodic boundary conditions. The crystal is assumed to be perfect and isotropic. Only the first nearest-neighbor interactions are considered. Since the crystal, subjected to a uniform temperature, undergoes a uniform thermal expansion, we use the change in lattice parameter as the only mechanical degree of freedom for the whole system. 


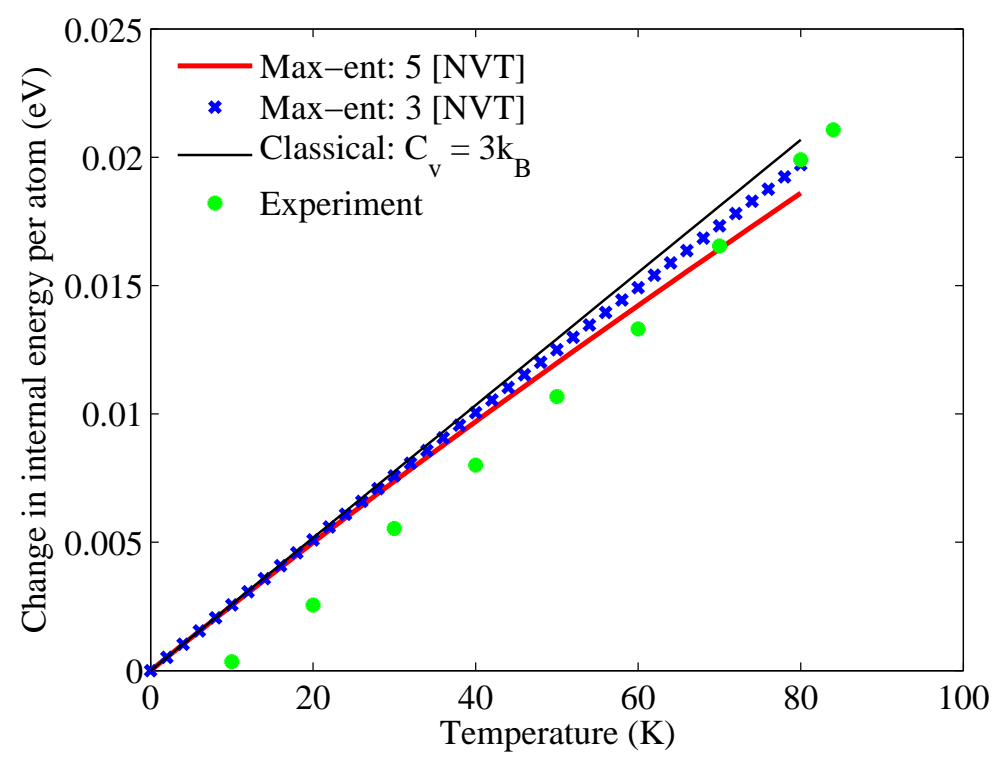

Figure 6.1: Change in internal energy of a perfect infinite Lennard-Jones crystal under NVT conditions based on the max-ent method using $3^{\text {rd }}$ and $5^{\text {th }}$ degree quadrature rules. The material used is solid Ar.

\subsection{Specific heat}

The specific heat of a system in thermal equilibrium at constant volume is defined as [28]

$$
C_{v}=\left.\frac{1}{N} \frac{\partial E}{\partial T}\right|_{V}
$$

where $N$ is the number of atoms in the crystal. $C_{v}$ is evaluated as the instantaneous slope of the internal energy versus temperature curve which is obtained by fixing the configuration at the initial state and equilibrating the system at the prescribed temperature $T$. The minimization problem becomes

$$
\min _{\omega \in \mathbb{R}} F\left(a_{0}, T, \omega\right) .
$$

The results are compared with experimental data [6] as well as the classical law of Dulong and Petit which yields

$$
C_{v}=3 k_{B}
$$

irrespective of the material. Figures 6.1 and 6.2 show that the max-ent predictions for $C_{v}$ are in very good agreement with the Dulong and Petit model at low and intermediate temperatures. The deviation from experimentally observed values at low temperature range is expected as the quantum mechanical effects dominate. As the temperature increases and approaches the melting temperature (Ar: $83 \mathrm{~K}, \mathrm{Cu}: 1337 \mathrm{~K}$ ), the anharmonic terms dominate and the predicted $C_{v}$ begins to deviate from $3 k_{B}$ as expected. Figure 6.1 also 


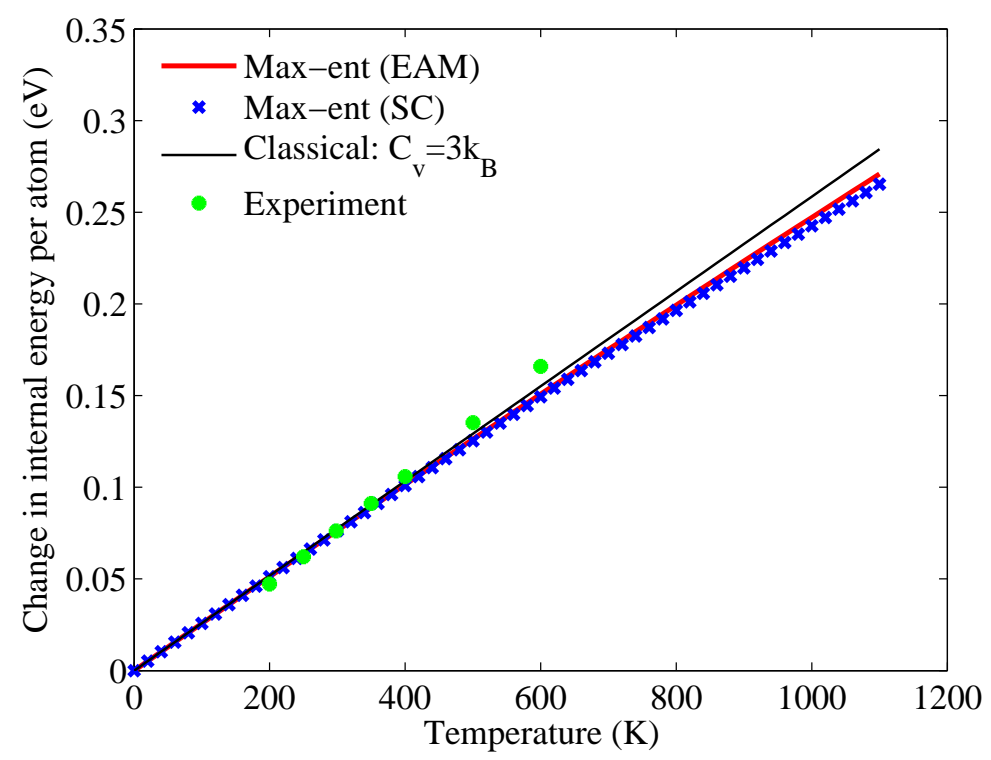

Figure 6.2: Change in internal energy of a perfect infinite $\mathrm{Cu}$ crystal under NVT conditions based on the max-ent method using Sutton-Chen and EAM-Johnson potentials.

shows that the results are consistent in the sense that the the $3^{\text {rd }}$ degree quadrature rule always predicts higher energy than the $5^{\text {th }}$ degree quadrature and is closer to the Dulong and Petit model. From figure 6.2, very little difference is observed between the results based on the EAM-Johnson potential and the Sutton-Chen potential.

\subsection{Thermal expansion}

The thermal expansion test is performed by prescribing a uniform temperature $T$ and equilibrating the sample under isothermal conditions. The minimization problem becomes

$$
\min _{a \in \mathbb{R}} \min _{\omega \in \mathbb{R}} F(a, T, \omega)
$$

where $a$ is the lattice parameter at temperature $T$. The minimization is performed using the non-linear version of the conjugate gradient method. The coefficient of linear thermal expansion is then given by the instantaneous slope of the thermal expansion versus temperature curve:

$$
\alpha(T)=\frac{\partial \varepsilon}{\partial T} \text { where } \varepsilon=\frac{a}{a_{0}}-1 .
$$

Figure 6.3 shows the thermal expansion of solid argon with temperature based on the max-ent approach using different quadrature rules and compared with MD simulations [29] and experimental data [21]. Since the max-ent method and molecular dynamics are both based on classical theory, they cannot capture the low temperature behavior, as observed in 


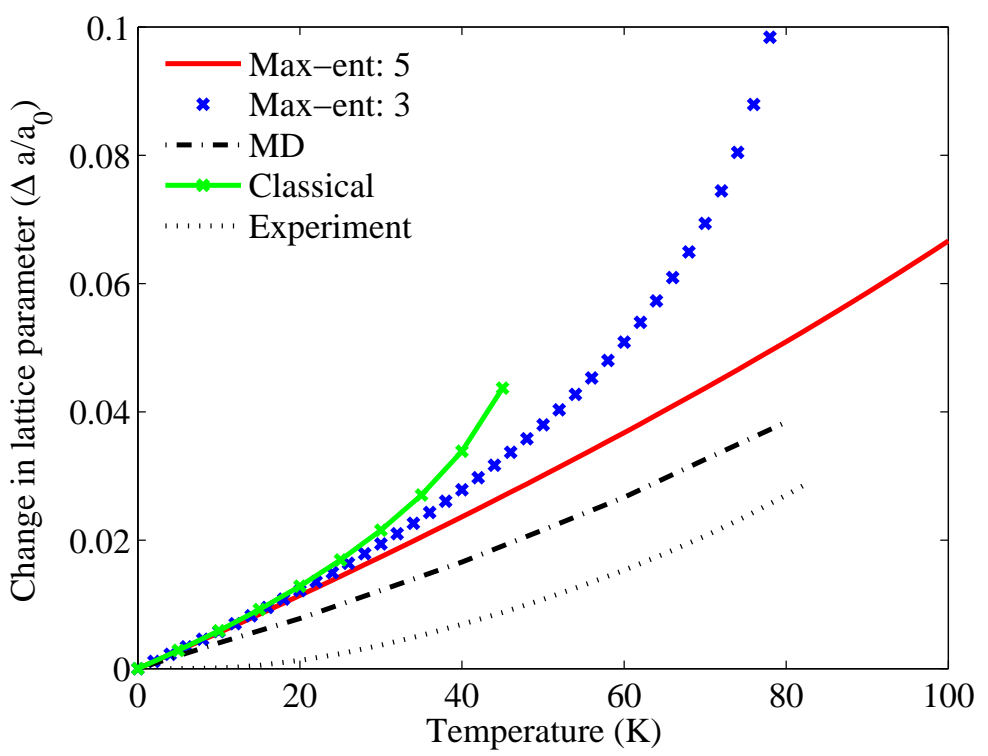

Figure 6.3: Thermal expansion of a perfect infinite Lennard-Jones crystal based on the maxent method using $3^{\text {rd }}$ and $5^{\text {th }}$ degree quadrature rules. The material used is solid Ar.

the experiments, which is governed by quantum effects. The results clearly indicate that the max-ent approach provides a significant improvement over the quasi-harmonic approximation with increasing degree of quadrature both in terms of accuracy as well as the range of temperature.

Figures 6.4 and 6.5 show the thermal expansion of $\mathrm{Cu}$ with temperature based on the Sutton-Chen potential and the EAM-Johnson potential, respectively, using $3^{\text {rd }}$ degree quadrature. The choice of the former was dictated by the availability of molecular dynamics results in the work of Çağin et al [1]. The experimental data for thermal expansion is shown only for $T>300$ where linear dependence on temperature is observed.

The max-ent curve in figure 6.4 shows a considerable improvement over the prediction of the local quasi-harmonic approximation at high temperatures and a good agreement with the MD results. However, since the results for the max-ent approach and molecular dynamics simulations are not in close agreement with experimental data, it is indicative of a limitation of the Sutton-Chen potential to model thermal properties rather than that of the proposed method. This is also observed by Çağin and coworkers [1]. On the contrary, figure 6.5 shows an excellent agreement of the max-ent results with experimental observations for the range of temperatures for which classical theory is valid [20]. This suggests that the EAM potential proposed by Johnson does better in predicting thermal properties than the potential proposed by Sutton and Chen.

Finally, we note that unlike in the case of the Lennard-Jones potential, the $3^{\text {rd }}$ degree quadrature works very well for the EAM-type potentials and hence suffices for the purpose of 


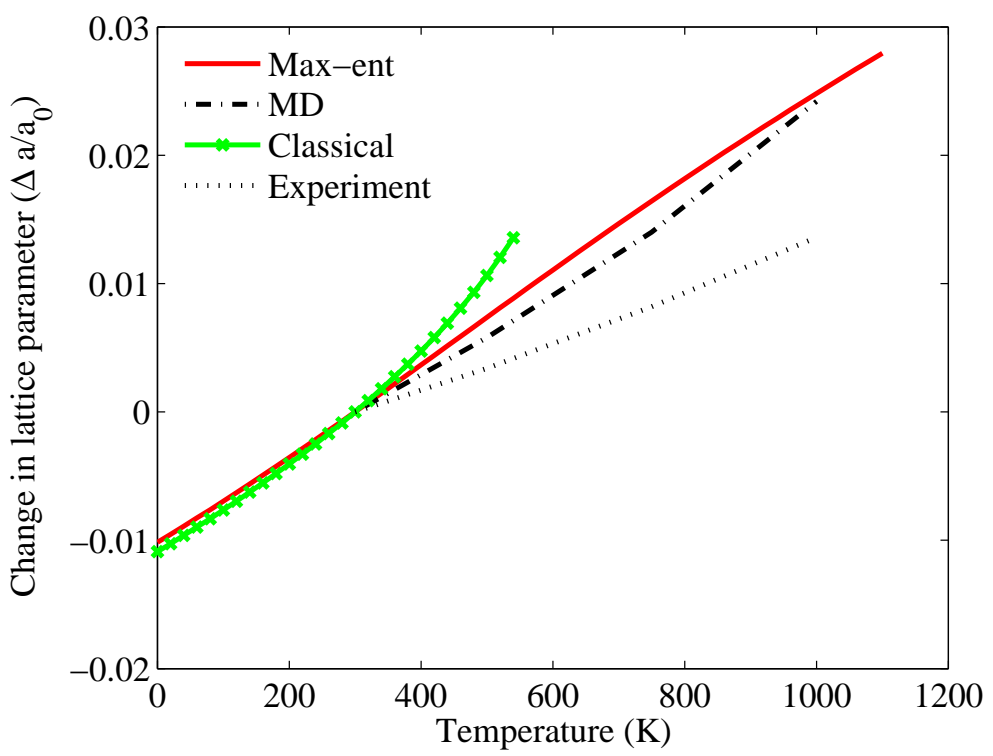

Figure 6.4: Thermal expansion of a perfect infinite $\mathrm{Cu}$ crystal based on the max-ent method with $3^{\text {rd }}$ degree quadrature. The results are based on the Sutton-Chen potential.

these calculations. This could, probably, be an effect of considering many-body interactions in the latter as opposed to pairwise interactions in the former. This is also suggested by the better agreement of EAM potential results with experiments and MD results as compared to those obtained using the Lennard-Jones potential.

This completes the overview of the max-ent approximation scheme. Although the method eliminates the dependence of the energy, $E(\overline{\boldsymbol{q}},\{S\},\{\omega\})$, on the microstate, the problem of determining the equilibrium configurations of the system still involves minimization over all atomistic degrees of freedom, $\overline{\boldsymbol{q}} \in \mathbb{R}^{3 N},\{S\} \in \mathbb{R}^{N}$, and $\{\omega\} \in \mathbb{R}^{N}$. We now seek to alleviate this task by coarse-graining the atomistic description in space using the framework of the quasicontinuum approach which we describe subsequently.

\section{Quasicontinuum method}

The chief objective of the quasicontinuum method is to systematically coarsen an atomistic description by the judicious introduction of kinematic constraints. These kinematic constraints are designed so as to preserve full atomistic resolution where required and to treat collectively large number of atoms in regions where the deformation field varies slowly on the scale of the lattice. Although different versions of the theory have been developed and documented [27, 19], here we review the three-dimensional version of the static quasicontinuum method developed by Knap and Ortiz [14] for zero temperature conditions, which we shall extend to the finite temperature case. 


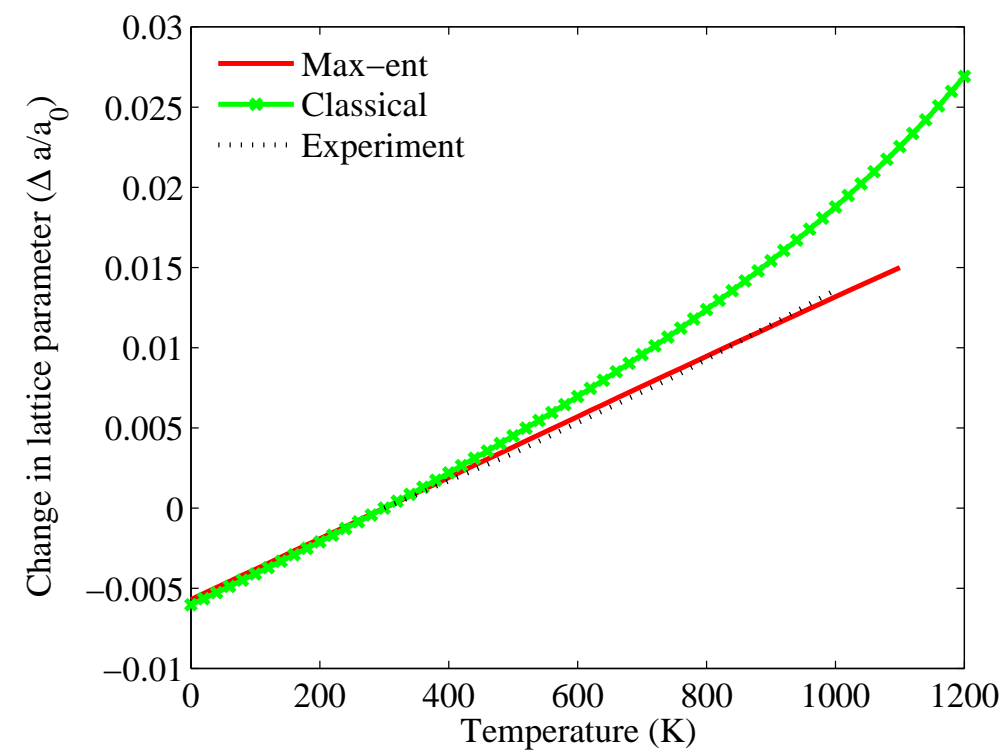

Figure 6.5: Thermal expansion of a perfect infinite $\mathrm{Cu}$ crystal based on the max-ent method with $3^{\text {rd }}$ degree quadrature. The results are based on the EAM-Johnson potential.

We consider a crystal with $N$ atoms in reference configuration occupying a subset $\mathscr{L}$ of a simple d-dimensional Bravais lattice. Denoting the basis vectors by $\left\{\boldsymbol{a}_{i} ; i=1, \ldots d\right\}$, the reference coordinates of the atoms are

$$
\boldsymbol{X}(\boldsymbol{l})=\sum_{i=1}^{d} l^{i} \boldsymbol{a}_{i}, \boldsymbol{l} \in Z \subset \mathbb{R}^{d} .
$$

$\boldsymbol{l}$ are the lattice coordinates associated with individual atoms, $Z$ is the set of integers, and $d$ is the dimension of space. We define $\boldsymbol{q} \in X \equiv \mathbb{R}^{N d}$ as the array of atomic positions in the deformed configuration, where $X$ denotes the configuration space of the crystal. We shall also use $\boldsymbol{q}(\boldsymbol{l}), \boldsymbol{l} \in \mathscr{L}$ to denote the coordinates of an individual atom.

At zero temperature, since the atoms do not exhibit thermal oscillations, the energy of the crystal is a function $E(\boldsymbol{q})$ expressed through the use of interatomic potentials. In the case of applied loads, we assume them to be conservative and to derive from an external potential $\Phi^{e x t}(\boldsymbol{q})$. Hence, the total potential energy is

$$
\Phi(\boldsymbol{q})=E(\boldsymbol{q})+\Phi^{e x t}(\boldsymbol{q}) .
$$

In addition, the crystal may be subjected to displacement boundary conditions over parts of its boundary. Then, the problem of determining the metastable equilibrium configurations of the system is a problem of seeking the local minima of the energy functional $\Phi(\boldsymbol{q})$, consistent with the essential boundary conditions. This may be stated as

$$
\min _{\boldsymbol{q} \in X} \Phi(\boldsymbol{q}) \text {. }
$$


For systems with a very large number of atoms, this minimization problem presents a significant computational burden. The essence of the theory of the quasicontinuum lies in replacing eq. (7.3) by an approximate minimization problem having the flexibility of preserving atomistic resolution in the regions of interest and treating atoms collectively where deformations are slow varying on the scale of the lattice. There are three key components of the quasicontinuum framework that impart the method its capabilities. We review these below.

\section{Constrained minimization}

The problem specified by eq. (7.3) is first replaced by a constrained minimization of $\Phi(\boldsymbol{q})$ over a suitably chosen subspace $X_{h}$ of $X . X_{h}$ is constructed by selecting a reduced set $\mathscr{L}_{h} \subset \mathscr{L}$ of $N_{h}<N$ representative atoms or nodes. The selection is done based on the local variation in the deformation field. Introducing a triangulation $\mathscr{T}_{h}$ over $\mathscr{L}_{h}$, the positions of the remaining atoms are determined by piecewise linear interpolation of the nodal coordinates

$$
\boldsymbol{q}_{h}(\boldsymbol{l})=\sum_{\boldsymbol{l}_{h} \in \mathscr{L}_{h}} \varphi\left(\boldsymbol{l} \mid \boldsymbol{l}_{h}\right) \boldsymbol{q}_{h}\left(\boldsymbol{l}_{h}\right)
$$

where $\varphi\left(\boldsymbol{l} \mid \boldsymbol{l}_{h}\right)$ denotes the continuous and piecewise linear shape function associated with the representative atom, $\boldsymbol{l}_{h} \in \mathscr{L}_{h}$, evaluated at the point $\boldsymbol{X}(\boldsymbol{l})$. Its domain is restricted to the simplices $K \in \mathscr{T}_{h}$ incident on $\boldsymbol{l}_{h}$, and it satisfies

$$
\begin{gathered}
\varphi\left(\boldsymbol{l}_{h}^{\prime} \mid \boldsymbol{l}_{h}\right)=\delta\left(\boldsymbol{l}_{h}^{\prime} \mid \boldsymbol{l}_{h}\right) \\
\sum_{\boldsymbol{l}_{h} \in \mathscr{L}_{h}} \varphi\left(\boldsymbol{l} \mid \boldsymbol{l}_{h}\right)=1
\end{gathered}
$$

where $\delta$ is the Dirac delta function. Eq. (7.5b) ensures that a constant field is interpolated exactly by the basis functions. The constrained minimization problem may now be stated as

$$
\min _{\boldsymbol{q}_{h} \in X_{h}} \Phi\left(\boldsymbol{q}_{h}\right) .
$$

The corresponding reduced equations of equilibrium are

$$
\boldsymbol{f}_{h}\left(\boldsymbol{l}_{h}\right)=\sum_{\boldsymbol{l} \in \mathscr{L}} \boldsymbol{f}(\boldsymbol{l}) \varphi\left(\boldsymbol{l} \mid \boldsymbol{l}_{h}\right)=\mathbf{0},
$$

where

$$
\boldsymbol{f}(\boldsymbol{l})=\frac{\partial \Phi}{\partial \boldsymbol{q}(\boldsymbol{l})}(\boldsymbol{q})
$$

\section{Sampling over clusters}

For a large crystal, performing full lattice sums as required in (7.7) is also an expensive computation. This difficulty is circumvented by sampling the behavior of the crystal over clusters 


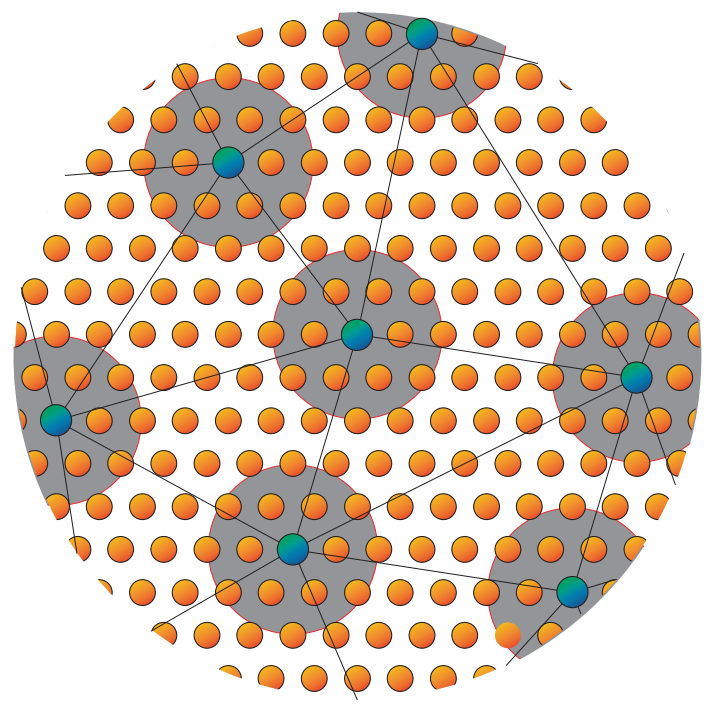

(a)

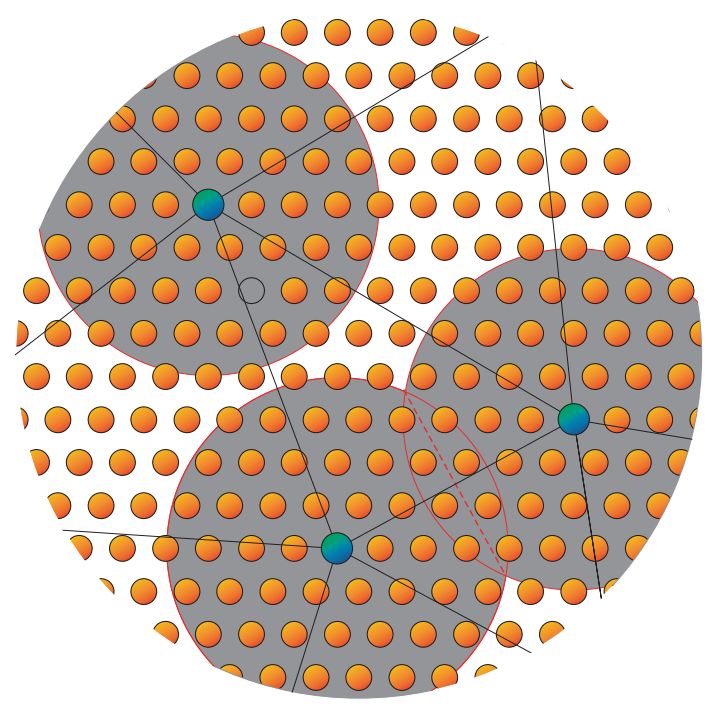

(b)

Figure 7.1: Clusters of atoms in the triangulation $\mathscr{T}_{h}$ of the crystal. a) Non-overlapping clusters. b) Merging of overlapping clusters.

of atoms around the representative atoms, as shown in Figure 7.1. This is demonstrated as follows. Let $\mathscr{C}\left(\boldsymbol{l}_{h}\right)$ be a cluster of lattice sites within a sphere of radius $r\left(\boldsymbol{l}_{h}\right)$ centered at the node $\boldsymbol{l}_{h}$. That is,

$$
\mathscr{C}\left(\boldsymbol{l}_{h}\right)=\left\{\boldsymbol{l}:\left|\boldsymbol{X}(\boldsymbol{l})-\boldsymbol{X}\left(\boldsymbol{l}_{h}\right)\right| \leq r\left(\boldsymbol{l}_{h}\right)\right\} .
$$

Let $g(\boldsymbol{l})$ be a lattice function whose sum over the lattice is

$$
S=\sum_{\boldsymbol{l} \in \mathscr{L}} g(\boldsymbol{l})
$$

Then, the cluster summation rule approximates $S$ by

$$
S \approx S_{h}=\sum_{\boldsymbol{l}_{h} \in \mathscr{L}_{h}} n_{h}\left(\boldsymbol{l}_{h}\right) S\left(\boldsymbol{l}_{h}\right),
$$

where $S\left(\boldsymbol{l}_{h}\right)$ denotes the sum over all atoms in the cluster $\mathscr{C}\left(\boldsymbol{l}_{h}\right)$, i.e.,

$$
S\left(\boldsymbol{l}_{h}\right)=\sum_{\boldsymbol{l} \in \mathscr{C}\left(\boldsymbol{l}_{h}\right)} g(\boldsymbol{l}) .
$$

The cluster weights $n_{h}\left(\boldsymbol{l}_{h}\right)$ associated with the nodes, $\boldsymbol{l}_{h} \in \mathscr{L}_{h}$, are computed by requiring that the cluster summation rule (7.11) be exact for all basis functions. Using eq. (7.11), the equations of equilibrium (7.7) are further reduced to the form

$$
\boldsymbol{f}_{h}\left(\boldsymbol{l}_{h}\right) \approx \sum_{\boldsymbol{l}_{h}^{\prime} \in \mathscr{L}_{h}} n_{h}\left(\boldsymbol{l}_{h}^{\prime}\right)\left[\sum_{\boldsymbol{l} \in \mathscr{C}\left(\boldsymbol{l}_{h}^{\prime}\right)} \frac{\partial F}{\partial \overline{\boldsymbol{q}}(\boldsymbol{l})} \varphi\left(\boldsymbol{l} \mid \boldsymbol{l}_{h}\right)\right]=\mathbf{0} .
$$


We wish to remark here that the cluster summation rules are so designed that the atomic forces are recovered exactly in regions with full atomistic resolution.

\section{Adaptive refinement}

The flexibility of the quasicontinuum method is further enhanced by the use of mesh adaption in order to tailor the computational mesh to the structure of the deformation field. Due to the lack of a rigorous theory relating the mesh size to suitable bounds on the energy for discrete systems, empirical adaption indicators based on the displacement field of the crystal are currently used. Specifically, the adaption indicator $\varepsilon(K)$ for simplex $K$ is measured as

$$
\varepsilon(K)=\sqrt{\left|I I_{E}(K)\right|} h(K)
$$

where $I I_{E}(K)$ is the second invariant of the Lagrangian strain tensor for simplex $K$, and $h(K)$ is the size of $K$. The element $K$ is deemed acceptable if

$$
\frac{\varepsilon(K)}{b}<T O L
$$

for some prescribed tolerance $T O L<1$ and is targeted for refinement otherwise. $b$ denotes the magnitude of the smallest Burgers vector of the crystal. Evidently, the value of TOL involves a compromise between conflicting demands on accuracy and computational efficiency. This summarizes the static theory of the quasicontinuum method. For further details and study of convergence characteristics, we refer the reader to [14].

\section{Extension to equilibrium thermodynamics}

As a precursor to the development of the full non-equilibrium finite temperature quasicontinuum method, we present here the framework for modelling equilibrium thermodynamic processes. One of the merits of our approach is that the finite temperature formulation for equilibrium problems possesses the same structure as the zero temperature quasicontinuum method, with the distinction that the energy functional to be minimized is no longer the potential energy given in eq. (7.2) but a temperature-dependent energy furnished by the max-ent method. Examples of systems in thermal equilibrium include systems undergoing thermal expansion at uniform temperature or subjected to quasistatic processes, such as nanoindentation or void growth under isothermal conditions. For such phenomena, we wish to determine the metastable configurations of the crystal when it is in thermal equilibrium at a uniform temperature $T$ under applied loads and boundary conditions. Based on the max-ent approximation scheme, the minimization problem may be enunciated as

$$
\min _{\overline{\boldsymbol{q}} \in X} \min _{\{\omega\} \in \mathbb{R}^{N}} \Phi(\overline{\boldsymbol{q}}, T,\{\omega\})
$$

with

$$
\Phi(\overline{\boldsymbol{q}}, T,\{\omega\})=F(\overline{\boldsymbol{q}}, T,\{\omega\})+\Phi^{e x t}(\overline{\boldsymbol{q}})
$$


$F$ being the Helmholtz free energy of the crystal derived earlier. We now seek to determine the solutions of eq. (8.1). As before, we first select a reduced set $\mathscr{L}_{h}$ of $N_{h}<N$ representative atoms based on the local variation of the displacement field. The positions, temperature, and approximate frequencies (mean field parameters) of the remaining atoms are interpolated over the nodes using piecewise linear shape functions defined before:

$$
\begin{aligned}
\overline{\boldsymbol{q}}_{h}(\boldsymbol{l}) & =\sum_{\boldsymbol{l}_{h} \in \mathscr{L}_{h}} \varphi\left(\boldsymbol{l} \mid \boldsymbol{l}_{h}\right) \overline{\boldsymbol{q}}_{h}\left(\boldsymbol{l}_{h}\right) \\
T_{h}(\boldsymbol{l}) & =\sum_{\boldsymbol{l}_{h} \in \mathscr{L}_{h}} \varphi\left(\boldsymbol{l} \mid \boldsymbol{l}_{h}\right) T_{h}\left(\boldsymbol{l}_{h}\right) \\
\omega_{h}(\boldsymbol{l}) & =\sum_{\boldsymbol{l}_{h} \in \mathscr{L}_{h}} \varphi\left(\boldsymbol{l} \mid \boldsymbol{l}_{h}\right) \omega_{h}\left(\boldsymbol{l}_{h}\right) .
\end{aligned}
$$

We must emphasize that since the mean field parameter $\omega(\boldsymbol{l})$ is an approximation for the average local frequency of that atom, the variation in $\{\omega\}$ follows the displacement field. This is because the local frequencies of an atom are computed from the eigenvalues of the local dynamical matrix associated with that atom, which in turn depends on the deformed configuration of the neighborhood of the atom. Therefore, in the regions where the displacement field is uniform, $\{\omega\}$ also varies slowly on the scale of the lattice, since all the atoms experience very similar environment. This provides a rationale for assuming the frequencies as a continuous field far from the atomistic domain and interpolating it over the representative atoms. The equilibrium equations are obtained by taking variations of the energy functional with respect to the nodal unknowns $\left(\overline{\boldsymbol{q}}_{h}\left(\boldsymbol{l}_{h}\right), \omega_{h}\left(\boldsymbol{l}_{h}\right)\right)$ and enforcing stationarity. The computational cost of solving the equilibrium equations can be further reduced by introducing cluster summation rules, as before. Thus, the final form of the equilibrium equations becomes

$$
\begin{aligned}
& \sum_{\boldsymbol{l}_{h}^{\prime} \in \mathscr{L}_{h}} n_{h}\left(\boldsymbol{l}_{h}^{\prime}\right)\left[\sum_{\boldsymbol{l} \in \mathscr{C}\left(\boldsymbol{l}_{h}^{\prime}\right)} \frac{\partial \Phi}{\partial \overline{\boldsymbol{q}}(\boldsymbol{l})} \varphi\left(\boldsymbol{l} \mid \boldsymbol{l}_{h}\right)\right]=\mathbf{0} \\
& \sum_{\boldsymbol{l}_{h}^{\prime} \in \mathscr{L}_{h}} n_{h}\left(\boldsymbol{l}_{h}^{\prime}\right)\left[\sum_{\boldsymbol{l} \in \mathscr{C}\left(\boldsymbol{l}_{h}^{\prime}\right)} \frac{\partial \Phi}{\partial \omega(\boldsymbol{l})} \varphi\left(\boldsymbol{l} \mid \boldsymbol{l}_{h}\right)\right]=0 .
\end{aligned}
$$

Finally, we require an appropriate adaption criterion in order to refine the mesh according to the deformation of the crystal while eliminating or reducing the refinement due to thermal expansion. To this end, we define $\varepsilon(K)$ as

$$
\varepsilon(K)=\sqrt{\left|I I_{E^{d}}(K)\right|} h(K),
$$

where $I I_{E^{d}}(K)$ is the second invariant of the deviatoric part of the Lagrangian strain tensor. The crystal is first allowed to expand freely at the prescribed temperature prior to indentation. The deformed configuration is then used as a reference configuration for computing $I I_{E^{d}}(K)$ during the subsequent load increments. 


\subsection{Convergence analysis}

In this section, we seek to understand the effect of coarsening on the energetics of the system by computing the error introduced by the quasicontinuum approximation. To this end, we consider a perfect crystal with periodic boundary conditions subjected to uniform temperature. We show that for a homogeneous deformation, the quasicontinuum approach gives the exact energy of the crystal irrespective of the degree of coarse-graining.

Theorem 8.1. Consider a perfect crystal with $N$ atoms and periodic boundary conditions occupying a subset $\mathscr{L}$ of a simple d-dimensional Bravais lattice. Let $\mathscr{L}_{h} \subset \mathscr{L}$ be a collection of $N_{h}<N$ representative atoms of the crystal. Then, for a homogeneous deformation under uniform temperature, the quasicontinuum energy is equal to the exact energy of the atomistic system. That is,

$$
\sum_{\boldsymbol{l}_{h} \in \mathscr{L}_{h}} n_{h}\left(\boldsymbol{l}_{h}\right)\left[\sum_{\boldsymbol{l} \in \mathscr{C}\left(\boldsymbol{l}_{h}\right)} E(\boldsymbol{l})\right]=\sum_{\boldsymbol{l} \in \mathscr{L}} E(\boldsymbol{l}) .
$$

Proof. When an infinite perfect crystal or a perfect crystal with periodic boundary conditions is subjected to uniform temperature or, more generally, a homogeneous deformation, every atom sees exactly the same environment and, consequently, has the same energy:

$$
E(\boldsymbol{l})=E_{1}, \quad \forall \boldsymbol{l} \in \mathscr{L} .
$$

Thus, the total internal energy of the system furnished by the max-ent approach becomes

$$
E(\overline{\boldsymbol{q}},\{S\},\{\omega\})=\sum_{\boldsymbol{l} \in \mathscr{L}} E(\boldsymbol{l})=N E_{1} .
$$

The quasicontinuum approximation of the total energy is obtained by using the cluster summation rule:

$$
\begin{aligned}
E_{h}\left(\boldsymbol{l}_{h}\right) & =\sum_{\boldsymbol{l}_{h} \in \mathscr{L}_{h}} n_{h}\left(\boldsymbol{l}_{h}\right) \sum_{\boldsymbol{l} \in \mathscr{C}\left(\boldsymbol{l}_{h}\right)} E(\boldsymbol{l}) \\
& =\sum_{\boldsymbol{l}_{h} \in \mathscr{L}_{h}} n_{h}\left(\boldsymbol{l}_{h}\right) N\left(\boldsymbol{l}_{h}\right) E_{1},
\end{aligned}
$$

where $N\left(\boldsymbol{l}_{h}\right)$ is the number of atoms in the cluster around node $\boldsymbol{l}_{h}$. We recall that the weights used in the cluster summation rules are determined such that the shape functions associated with all the nodes are summed exactly. That is,

$$
\sum_{\boldsymbol{l}_{h}^{\prime} \in \mathscr{L}_{h}} n_{h}\left(\boldsymbol{l}_{h}^{\prime}\right) \sum_{\boldsymbol{l} \in \mathscr{C}\left(\boldsymbol{l}_{h}^{\prime}\right)} \varphi\left(\boldsymbol{l} \mid \boldsymbol{l}_{h}\right)=\sum_{\boldsymbol{l} \in \mathscr{L}} \varphi\left(\boldsymbol{l} \mid \boldsymbol{l}_{h}\right), \quad \forall \boldsymbol{l}_{h} \in \mathscr{L}_{h} .
$$

Summing both sides over $\boldsymbol{l}_{h} \in \mathscr{L}_{h}$, rearranging the sums, and using the property

$$
\sum_{\boldsymbol{l}_{h} \in \mathscr{L}_{h}} \varphi\left(\boldsymbol{l} \mid \boldsymbol{l}_{h}\right)=1
$$


eq. (8.9) reduces to

$$
\sum_{\boldsymbol{l}_{h}^{\prime} \in \mathscr{L}_{h}} n_{h}\left(\boldsymbol{l}_{h}^{\prime}\right) N\left(\boldsymbol{l}_{h}\right)=N
$$

Multiplying eq. (8.11) by $E_{1}$ and comparing the expressions with eq. (8.7) and eq. (8.8b), we conclude that

$$
E_{h}=E
$$

and that this equality of energies is independent of the degree of coarsening.

As a concluding remark, we note that this result is valid for infinite crystals and that for finite crystals, the surface effects should introduce some error. Nevertheless, the result demonstrates that in the regions within the bulk of the crystal and away from defects, the atoms experience similar environments and deformations and, consequently, the quasicontinuum energy should be a very good approximation.

\section{Variational formulation for thermo-mechanical cou- pled problems}

We now present a review of a variational formulation for coupled thermo-mechanical boundaryvalue problems for general dissipative solids proposed by Yang et al. in [30], and which we shall use to model heat conduction using finite temperature quasicontinuum method.

The aim of this work is to characterize variationally the solutions of equilibrium problems for an inelastic deformable solid capable of conducting heat. Specifically, the work shows the existence of a joint potential function whose Euler-Lagrange equations yield the equilibrium equations, the kinetic relations, and the conservation of energy. A general dissipative solid may be understood as a deformable solid, undergoing large deformations, possessing viscosity and internal processes, and conducting heat. However, in the context of the current work, we shall restrict this review to conducting thermoelastic solids. The only kinetic relation that we introduce a priori is the Fourier's law of heat conduction.

We consider a body occupying a region $B \subset \mathbb{R}^{3}$ in reference configuration and undergoing a thermodynamic process. The motion of the body is described by a time-dependent deformation mapping $\varphi: B \times[a, b] \rightarrow \mathbb{R}^{3}$, where $[a, b]$ is the time interval of the motion. The body may be subjected to essential boundary conditions for the displacement and temperature over parts of its boundary. Let $\bar{H}$ be the prescribed outward heat flux on the Neumann boundary $\partial_{N} B$, and let $\overline{\boldsymbol{T}}$ be the applied traction on the traction boundary $\partial_{T} B$. We assume that there exists an internal energy density expressed as a function of the local state,

$$
E=E(\boldsymbol{F}, S),
$$

where $\boldsymbol{F}=\operatorname{Grad} \varphi$ is the deformation gradient, and $S$ is the local entropy density per unit undeformed volume. Then, the equilibrium stress is given by

$$
\boldsymbol{P}^{e} \equiv \partial_{\boldsymbol{F}} E(\boldsymbol{F}, S)
$$


and the local equilibrium temperature is given by the relation

$$
\Theta=\partial_{S} E=E(\boldsymbol{F}, S)
$$

For the variational formulation, it is necessary to differentiate between the equilibrium temperature $\Theta$ and an external temperature field $T$. Although they are equal everywhere at equilibrium, the condition is not imposed a priori. We also assume that a Fourier potential $\chi(\boldsymbol{G})$ exists such that

$$
\boldsymbol{H}=\partial_{\boldsymbol{G}} \chi(\boldsymbol{G}),
$$

where $\boldsymbol{H}$ is the heat flux, and $\boldsymbol{G}=-T^{-1} \operatorname{Grad} T$. $\chi$ is assumed to be quadratic and strictly convex in $G$, which guarantees a unique minimum.

\section{Variational formulation}

A rate problem is understood as a problem of finding the rate of change of the state of the body given its current state and appropriate forcing and boundary conditions. For a thermoelastic problem, this means the problem of determining $(T, \dot{S})$ given the current local state $(\boldsymbol{F}, S)$. To this end, we construct a joint potential function of the following form:

$$
\begin{aligned}
\Phi[T, \dot{S}] & =\int_{B}[(\Theta-T) \dot{S}-\chi(\boldsymbol{G})] d V \\
& +\int_{B} R Q \log \frac{T}{T_{0}} d V-\int_{\partial_{N} B} \bar{H} \log \frac{T}{T_{0}} d S,
\end{aligned}
$$

where $Q$ is the distributed heat source per unit mass, and $T_{0}$ is a reference temperature. Then, according to [30], the problem of determining solutions for the thermoelastic rate problem may be stated as a two field variational problem:

$$
\inf _{\dot{S}} \sup _{T} \Phi[T, \dot{S}] .
$$

Taking variations of this potential with respect to the fields $(T, \dot{S})$ and enforcing stationarity yields the thermoelastic rate problem in strong form:

$$
\begin{array}{lr}
T \dot{S}=-\operatorname{Div} \boldsymbol{H}+R Q & \text { in } B, \\
\boldsymbol{H} \cdot \boldsymbol{N}=\bar{H} & \text { on } \partial_{N} B, \\
T=\bar{T} & \text { on } \partial B \backslash \partial_{N} B, \\
T=\Theta & \text { in } B .
\end{array}
$$

Thus, the general rate problem for thermoelastic conducting solids is equivalent to the stationarity principle:

$$
\delta \Phi=0 .
$$




\section{Incremental formulation}

We now present a time-discretized version of the variational problem as established in [30]. The purpose of time-discretization is to reduce the modelling of time-dependent phenomena to a sequence of incremental problems, each characterized by a variational principle. For a rigorous derivation of the variational updates, we refer the reader to [30]. Below, we give an outline of the incremental extremum problem formulated by identifying a convenient joint potential which is consistent with the field equations. To this end, we consider a sequence of times $t_{0}, \ldots, t_{n} \ldots$ and seek to characterize the state $(\varphi, T, S)$ of the solid at those times. Specifically, we wish to determine approximately the state $\left(\varphi_{n+1}, T_{n+1}, S_{n+1}\right)$ at $t_{n+1}$ assuming that the state $\left(\varphi_{n}, T_{n}, S_{n}\right)$ is known. We construct a family of incremental potentials based on the backward Euler finite difference scheme:

$$
\begin{aligned}
& \Phi_{n}\left[\varphi_{n+1}, T_{n+1}, S_{n+1}\right]= \\
& \int_{B}\left[E_{n+1}-E_{n}-T_{n+1}\left(S_{n+1}-S_{n}\right)-\Delta t \chi_{n+1}\right] d V \\
& -\int_{B} R \boldsymbol{B}_{n+1} \cdot\left(\varphi_{n+1}-\varphi_{n}\right) d V-\int_{\partial_{T} B} \overline{\boldsymbol{T}}_{n+1} \cdot\left(\varphi_{n+1}-\varphi_{n}\right) d S \\
& +\int_{B} \triangle t R Q_{n+1} \log \frac{T_{n+1}}{T_{n}} d V-\int_{\partial_{N} B} \Delta t \bar{H}_{n+1} \log \frac{T_{n+1}}{T_{n}} d S,
\end{aligned}
$$

with

$$
\chi_{n+1}=\chi\left(\boldsymbol{G}_{n+1}\right), \boldsymbol{G}_{n+1}=-\operatorname{Grad} \log \frac{T_{n+1}}{T_{n}} .
$$

Then the incremental variational problem becomes

$$
\inf _{\varphi_{n+1}} \inf _{S_{n+1}} \sup _{T_{n+1}} \Phi_{n}\left[\varphi_{n+1}, T_{n+1}, S_{n+1}\right] .
$$

Taking variations and enforcing stationarity as before yields the equilibrium equation, the heat equation, and the natural boundary conditions:

$$
\begin{array}{lr}
\operatorname{Div} \boldsymbol{P}_{n+1}^{e}+R \boldsymbol{B}_{n+1}=O(\triangle t) & \text { in } B, \\
\boldsymbol{P}_{n+1}^{e} \cdot \boldsymbol{N}-\overline{\boldsymbol{T}}_{n+1}=O(\triangle t) & \text { on } \partial_{T} B, \\
\frac{S_{n+1}-S_{n}}{\triangle t}=-\frac{1}{T_{n+1}} \operatorname{Div} \partial_{\boldsymbol{G}_{n+1}} \chi_{n+1}+\frac{1}{T_{n+1}} R Q_{n+1} & \text { in } B, \\
\partial_{\boldsymbol{G}_{n+1}} \chi_{n+1} \cdot \boldsymbol{N}=\bar{H}_{n+1} & \text { on } \partial_{N} B, \\
\partial_{S_{n+1}} E_{n+1}-T_{n+1}=O(\triangle t) & \text { in } B .
\end{array}
$$

All field equations are recovered as $\Delta t \rightarrow 0$. Thus, the time-discretized variational formulation for dissipative solids provides a means of reducing the rate problem to an incremental problem with a variational structure. Moreover, the incremental potential $\Phi_{n}$ reflects both the energetics as well as the kinetics of the material. This time-discretization of the rate problem provides a way of incorporating the heat equation into the finite temperature quasicontinuum framework that was presented in section 8 . 


\section{Quasicontinuum method and heat transport}

In this section, we develop a full three-dimensional non-equilibrium finite temperature version of the quasicontinuum theory based on the max-ent approximation scheme. Following the review in the previous section, we construct an incremental potential based on the backward Euler finite difference scheme as

$$
\begin{aligned}
& \Phi_{n}\left[\overline{\boldsymbol{q}}_{n+1},\left\{T_{n+1}\right\},\left\{S_{n+1}\right\},\left\{\omega_{n+1}\right\}\right]= \\
& \left(E_{n+1}-E_{n}\right)-\sum_{\boldsymbol{l} \in \mathscr{L}} T_{n+1}(\boldsymbol{l})\left[S_{n+1}(\boldsymbol{l})-S_{n}(\boldsymbol{l})\right]+\int_{B} \Delta_{n+1}\left(\boldsymbol{G}_{n+1}(\boldsymbol{X})\right) \triangle t d V \\
& +\int_{B} \triangle t R Q_{n+1} \log \frac{T_{n+1}}{T_{n}} d V-\int_{\partial_{N} B} \triangle t \bar{H}_{n+1} \log \frac{T_{n+1}}{T_{n}} d S,
\end{aligned}
$$

where $\triangle t$ is the time step, $\boldsymbol{G}_{n+1}=-T_{n+1}^{-1} \operatorname{Grad} T_{n+1}$, and $E_{n+1}$ is the global internal energy of the crystal at $t=(n+1) \triangle t$, furnished by the max-ent scheme. $B \subset \mathbb{R}^{3}$ denotes the region occupied by the crystal in the reference configuration. $\bar{H}$ denotes the outward heat flux prescribed on the Neumann boundary $\partial_{N} B . Q_{n+1}$ is the local heat generated at time $t_{n+1}$. In the current work, we neglect any heat sources and hence assume $Q_{n+1}=0$. For general dissipative solids, $\Delta$ may be a kinetic potential which gives the viscosity law, rate sensitivity, and heat conduction. In the context of this work,

$$
\Delta=-\chi,
$$

where $\chi$ is a Fourier potential. As before, $\chi$ is assumed to be strictly convex and quadratic in $\boldsymbol{G}$. We also assume the Fourier law of heat conduction, which furnishes a linear dependence of the heat flux on the temperature gradient at that point:

$$
\partial_{G} \Delta_{n+1}=-\boldsymbol{H}_{n+1}=\kappa \operatorname{Grad} T_{n+1}(\boldsymbol{l}) .
$$

We wish to note that the Fourier law is a phenomenological relation and, therefore, introduces the heat conductivity, $\kappa$, as an empirical parameter into the model in addition to the empirical interatomic potential. The incremental variational problem may be enunciated as

$$
\inf _{\overline{\boldsymbol{q}}_{n+1}} \inf _{\left\{S_{n+1}\right\}} \inf _{\left\{\omega_{n+1}\right\}} \sup _{\left\{T_{n+1}\right\}} \Phi_{n}\left[\overline{\boldsymbol{q}}_{n+1},\left\{T_{n+1}\right\},\left\{S_{n+1}\right\},\left\{\omega_{n+1}\right\}\right] .
$$

The temperature field and $\{\omega\}$ can take only positive values. Therefore, for the purpose of numerical implementation, we recast the problem in terms of new fields defined as

$$
\begin{aligned}
v_{n+1}(\boldsymbol{l}) & =\log \frac{T_{n+1}(\boldsymbol{l})}{T_{n}(\boldsymbol{l})} \\
\mu_{n+1}(\boldsymbol{l}) & =\log \frac{\omega_{n+1}(\boldsymbol{l})}{\omega_{n}(\boldsymbol{l})}
\end{aligned}
$$


and which can take values on the entire real line $\mathbb{R}$. We follow the same procedure as presented in sections 7 and 8 to constrain the extremum problem over representative atoms. As before, we introduce interpolations over nodal variables. Eq. (8.3a) remains the same while eq. (8.3c) and eq. (8.3b) are replaced by

$$
\begin{aligned}
& v_{n+1}^{h}(\boldsymbol{l})=\sum_{\boldsymbol{l}_{h} \in \mathscr{L}_{h}} \varphi\left(\boldsymbol{l} \mid \boldsymbol{l}_{h}\right) v_{n+1}^{h}\left(\boldsymbol{l}_{h}\right) \\
& \mu_{n+1}^{h}(\boldsymbol{l})=\sum_{\boldsymbol{l}_{h} \in \mathscr{L}_{h}} \varphi\left(\boldsymbol{l} \mid \boldsymbol{l}_{h}\right) \mu_{n+1}^{h}\left(\boldsymbol{l}_{h}\right) .
\end{aligned}
$$

Finally, we take variations of the incremental energy functional, $\Phi_{n}$, with respect to the nodal unknowns. Enforcing stationarity of the incremental potential yields the following equilibrium equations:

$$
\begin{aligned}
& \sum_{\boldsymbol{l}_{h}^{\prime} \in \mathscr{L}_{h}} n_{h}\left(\boldsymbol{l}_{h}^{\prime}\right)\left[\sum_{\boldsymbol{l} \in \mathscr{C}\left(\boldsymbol{l}_{h}^{\prime}\right)} \frac{\partial E_{n+1}}{\partial \overline{\boldsymbol{q}}_{n+1}(\boldsymbol{l})} \varphi\left(\boldsymbol{l} \mid \boldsymbol{l}_{h}\right)\right]=\mathbf{0} \\
& \sum_{\boldsymbol{l}_{h}^{\prime} \in \mathscr{L}_{h}} n_{h}\left(\boldsymbol{l}_{h}^{\prime}\right)\left[\sum_{\boldsymbol{l} \in \mathscr{C}\left(\boldsymbol{l}_{h}^{\prime}\right)}\left(\frac{\partial E_{n+1}}{\partial S_{n+1}(\boldsymbol{l})}-T_{n+1}(\boldsymbol{l})\right) \varphi\left(\boldsymbol{l} \mid \boldsymbol{l}_{h}\right)\right]=0 \\
& \sum_{\boldsymbol{l}_{h}^{\prime} \in \mathscr{L}_{h}} n_{h}\left(\boldsymbol{l}_{h}^{\prime}\right)\left[\sum_{\boldsymbol{l} \in \mathscr{C}\left(\boldsymbol{l}_{h}^{\prime}\right)} \frac{\partial E_{n+1}}{\partial \mu_{n+1}(\boldsymbol{l})} \varphi\left(\boldsymbol{l} \mid \boldsymbol{l}_{h}\right)\right]=0
\end{aligned}
$$

and the energy balance equation:

$$
\begin{aligned}
& -\sum_{\boldsymbol{l}_{h}^{\prime} \in \mathscr{L}} n_{h}\left(\boldsymbol{l}_{h}^{\prime}\right)\left[\sum_{\boldsymbol{l} \in \mathscr{C}\left(\boldsymbol{l}_{h}^{\prime}\right)}\left(S_{n+1}(\boldsymbol{l})-S_{n}(\boldsymbol{l})\right) T_{n}(\boldsymbol{l}) \exp \left[v_{n+1}(\boldsymbol{l})\right] \varphi\left(l \mid l_{h}\right)\right] \\
& -\int_{B} \kappa \Delta t\left[\operatorname{Grad} T_{n}(\boldsymbol{X})+T_{n}(\boldsymbol{X}) \operatorname{Grad} v_{n+1}(\boldsymbol{X})\right] \exp \left[v_{n+1}(\boldsymbol{X})\right] \operatorname{Grad} \varphi\left(\boldsymbol{X} \mid l_{h}\right) d V \\
& -\int_{\partial_{N} B} \triangle t \bar{H}_{n+1} \varphi\left(\boldsymbol{X} \mid \boldsymbol{l}_{h}\right) d S=0 .
\end{aligned}
$$

If external loads are applied, we assume them to be conservative as before, and the energy functional $E$ in eq. (10.6a) is replaced by

$$
E\left(\overline{\boldsymbol{q}}_{n+1},\left\{S_{n+1}\right\},\left\{\omega_{n+1}\right\}\right)+\Phi^{e x t}(\overline{\boldsymbol{q}}) .
$$

We recall from section 4 that enforcing the equipartition of energy and using the relation $\sigma^{2}(\boldsymbol{l})=k_{B} T(\boldsymbol{l})$ yields a local equilibrium relation between entropy and temperature:

$$
S_{n+1}(\boldsymbol{l})=3 k_{B} \log \frac{k_{B} T_{n+1}(\boldsymbol{l})}{\hbar \omega_{n+1}(\boldsymbol{l})}+4 k_{B}-k_{B} \log N,
$$


and we use it to substitute for $S_{n+1}(\boldsymbol{l})$ in the equations above. Consequently, we do not have to solve eq. (10.6b). Nevertheless, we do evaluate the left-hand side of eq. (10.6b) in all our numerical tests and confirm that eq. (10.6b) is satisfied automatically. We also observe from eq. (10.7) that the rate term of the heat equation is computed using the cluster summation rule of the quasicontinuum method, whereas the diffusion term in the heat equation is an integral and is evaluated using Gauss quadrature as in a finite element analysis [9].

Before concluding this section, we wish to emphasize that the backward Euler finite difference scheme used in eq. (10.1) is unconditionally stable and hence does not impose any limitation on the time-step, $\Delta t$. Nevertheless, an estimate of the critical time-step can be obtained as

$$
\Delta t_{c} \approx \frac{h^{2}}{D}
$$

where $D$ is the diffusion constant for the material. $h$ is the element size and, in the regions with atomistic resolution, is equal to the lattice spacing. This implies that for a coarsegrained system with a smooth temperature gradient, the critical time-step would increase as the square of the element size. Consequently, larger time-steps may be used allowing the simulation of slow processes as well. This is unlike molecular dynamics which is limited to a time-step of about $10^{-14}$ seconds, which is of the order of the time-period of the thermal oscillations of the atoms.

\section{Numerical tests and discussion}

In this section, we summarize and discuss our results for numerical tests that demonstrate the applicability of the non-equilibrium finite temperature quasicontinuum method to modelling thermodynamic phenomena. We take nanoindentation as a convenient test problem as it is a conventional experimental tool for studying complex material behavior such as microstructure evolution via defect nucleation and propagation. Here we present the results for finite temperature nanoindentation tests performed with a rectangular punch for three different indenter velocities. This problem has been studied as a plane strain problem using static QC by Shenoy et al [23]. The purpose of the tests is two-fold. First, they serve to illustrate the ability of our method to simulate processes ranging from isothermal or very slow to adiabatic or very fast (although slow enough for inertial effects to be negligible). Second, they also enable us to understand the effect of the indentation velocity on the evolution of the temperature distribution.

\section{Test problem definition}

The test sample is an fcc nearest-neighbor Lennard-Jones crystal with $32 \times 32 \times 32$ unit cells, or a total of 137,313 atoms. Solid Argon is used as a test material since it can be modelled using the Lennard-Jones pair potential. The surfaces of the sample are aligned with the cube directions (Figure 11.1). The indenter is a rigid rectangular indenter and is applied along one of the diagonals of the top surface of the cube, as shown in Figure 11.2 


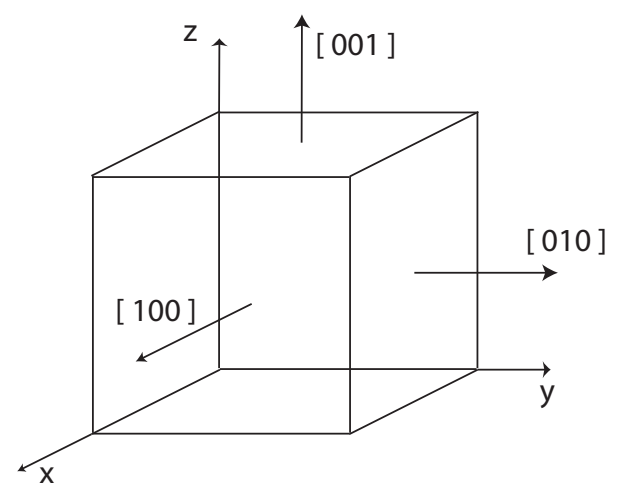

Figure 11.1: Crystallographic orientation of the test sample used in the simulations.

since the (111) planes are the preferred slip planes for an fcc crystal. ABCD corresponds to the (110) plane. The initial mesh comprises of atomistic region only in the vicinity of the indenter and has 3506 representative atoms. Figure 11.3 shows the cross-section ABCD with the initial triangulation. The boundary conditions are imposed as follows. In order to allow free thermal expansion, only the z-displacement of the nodes on the bottom surface is constrained. Using the symmetry of the problem, the node at the center of the bottom surface is fixed completely in order to avoid rigid motion. No constraint is applied on the lateral surfaces. The indenter is applied as a displacement boundary condition on a band of atoms on the top surface corresponding to the width of the punch. In our calculations, we used the following values for the width of the punch, $W$, and the incremental indentation depth, $\delta$ :

$$
W=9[\sigma], \quad \delta=-0.1[\sigma]
$$

where $\sigma$ is the nearest-neighbor distance for an fcc crystal $(\sigma=0.34 \mathrm{~nm}$ for Ar). The length of the face diagonal is $64[\sigma]$. Before indentation, the cube is allowed to equilibrate isothermally at a uniform temperature of $T=0.5 T_{m}, T_{m}$ being the melting temperature $\left(T_{m}=83 \mathrm{~K}\right.$ for solid $\left.\mathrm{Ar}\right)$.

The first test is performed under adiabatic conditions by imposing $\triangle t=0$. Simply put, it means that the indentation occurs so fast that there is no time for the generated heat to dissipate. We wish to remark here that this is a qualitative result since the inertial effects should also be taken into account for very fast processes. The second test is performed with $\triangle t=50\left[\kappa \sigma / k_{B}\right]$, which is equivalent to a time step of $17.4 \mathrm{ps}$. The indenter velocity may be calculated as

$$
V=\frac{\delta}{\triangle t}=1.95 \mathrm{~m} / \mathrm{s}
$$

The third test is performed with $\triangle t=5\left[\kappa \sigma / k_{B}\right]$ which corresponds to an indentation velocity of $19.5 \mathrm{~m} / \mathrm{s}$. 


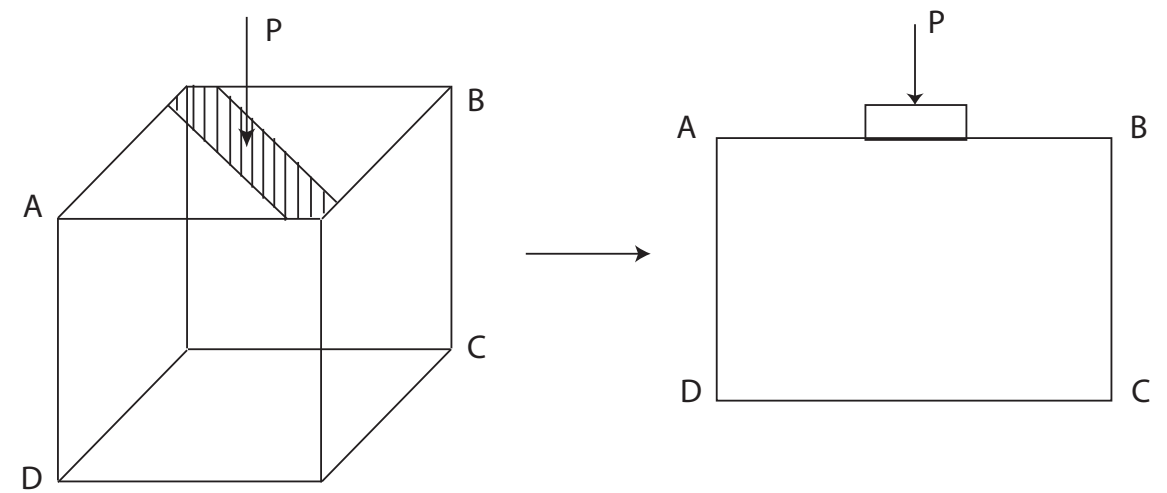

Figure 11.2: Geometry of the nanoindentation setup for a rectangular indenter.

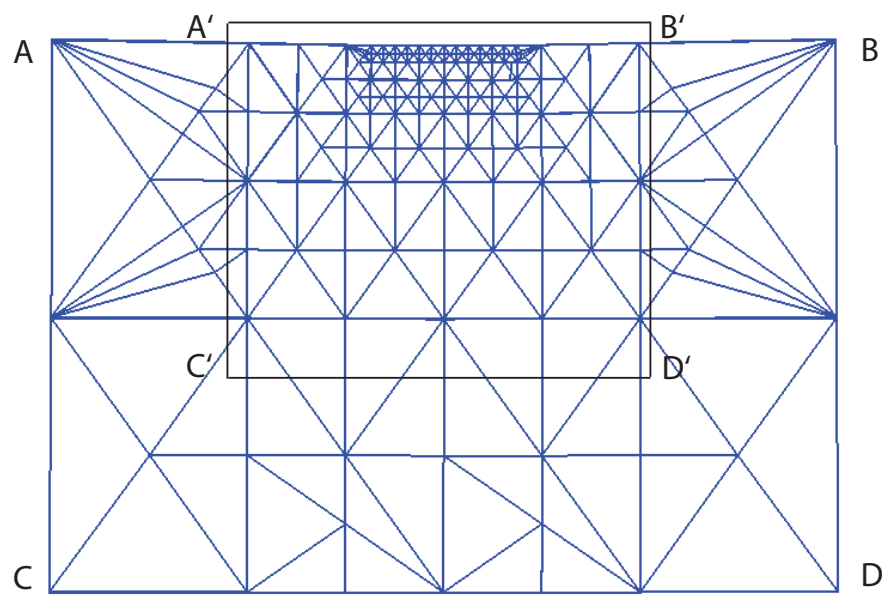

Figure 11.3: Initial mesh for the nanoindentation test with rectangular indenter. A'B'C'D' is the region shown in the snapshots of the temperature evolution.

\section{Results and discussion}

Figures 11.5 and 11.6 show the snapshots of the temperature profile under the indenter for the adiabatic test. Figures 11.7 and 11.8 present images of the temperature profile for the second and third tests. Figure 11.4 presents a comparison of the force versus indenter depth curves for the two cases with very fast and very slow indentation velocities.

Based on Figures 11.5 and 11.6, we observe the evolution of temperature as the indentation proceeds in the adiabatic case. In the last image in Figures 11.6, when the indenter depth is $-1.2[\sigma]$, the temperature under the edges of the punch is $0.6 T_{m}$, which is a $20 \%$ rise from the prescribed temperature. The heating under the edges of the indenter is expected due to high stresses. In addition, we note that this hot region spreads out along the (111) plane as the dislocation propagates. However, the significant temperature difference at the 


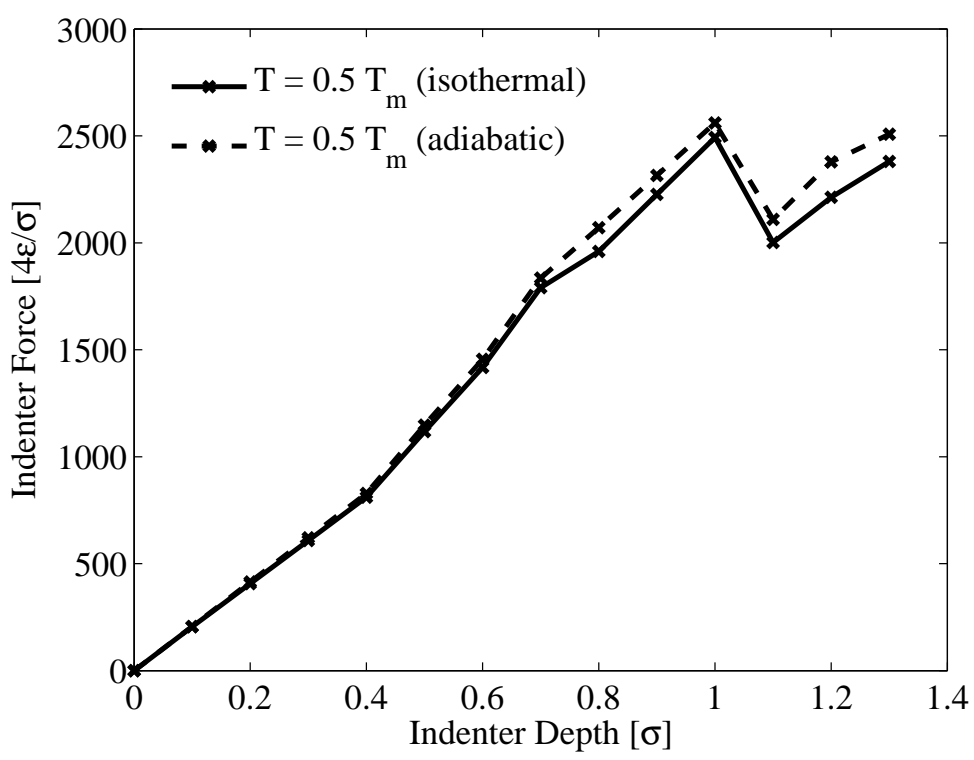

Figure 11.4: Force versus indenter depth plot for a rectangular indenter using the max-ent approach under adiabatic and isothermal conditions.

dislocation core needs further investigation. It is probable that the temperature difference is mediated by the deformation field above and below the slip plane. Furthermore, since the test is adiabatic, the system is unable to dissipate the heat generated.

Figure 11.4 indicates a negligible variation in the force indenter curve for the adiabatic and isothermal cases. We also note that the dislocation nucleation occurs at the same indenter depth for both speeds. Furthermore, the drop in the force is much more pronounced due to higher stress concentration caused by the sharp edges.

Figure 11.7 shows the temperature profile under the indenter for the second test with heat conduction. The negligible change in temperature implies that the indentation speed is slow enough to allow dissipation of the heat generated locally. In other words, the time step used in the test is such that the indenter velocity is less than the critical velocity for the material, thereby making the process isothermal. We use the diffusivity, $D$, and the lattice constant of solid Ar to evaluate the critical velocity as

$$
V_{c}=\frac{D}{a}=17.3 \mathrm{~m} / \mathrm{s}
$$

which confirms that the indentation velocity, $V<V_{c}$. We wish to emphasize that the time step of 17.4 ps is about 1800 times larger than a typical time step used molecular dynamics simulations. This demonstrates that the proposed method for modelling non-equilibrium phenomena using the quasicontinuum framework does not impose any limitations on the time step. Thereby, it facilitates the simulation of very slow thermodynamic processes which are not accessible to atomistic models. 
In both these tests, which use a mesh refinement tolerance TOL $=0.008$, it is observed that the stacking fault ends where the coarse-grained region begins, which may be a potential source of numerical error. This limitation may be rectified by lowering the refinement tolerance TOL and by allowing the system to relax and remesh at the same load.

The third test is performed with a stricter tolerance for mesh refinement $(\mathrm{TOL}=0.004)$. Consequently, we observe heavier remeshing and almost full atomistic resolution is achieved by the time a dislocation nucleates. We would like to emphasize here that since all the representative atoms are inserted in the vicinity of the indenter, the ratio of nodes to the total number of atoms can be made arbitrarily small by considering larger samples.

It is also observed in this test that the nucleation occurs at $\delta=-1.2[\sigma]$ which is delayed compared to the previous tests wherein the nucleation occurs at $\delta=-1.0[\sigma]$. Clearly, this indicates that there should be an optimal value of TOL that would strike a balance between accuracy and performance demands. Finally, the small variation in the temperature distribution in the sample is in agreement with the previous tests and is expected since the indentation velocity lies very close to the critical velocity.

Figure 11.9 shows the dislocation structure under a rectangular indenter extracted using the centerosymmetry parameter [13]. As expected, we observe that the first partial dislocation is emitted along the (111) plane followed by a stacking fault ribbon (Figure 11.10). Since the nearest-neighbor Lennard-Jones potential has zero stacking fault energy, we do not observe a second partial dislocation and the stacking fault continues to grow in size as the indentation proceeds.

\section{Concluding remarks}

In this paper, we have developed a variational approach to coarse-graining of equilibrium and non-equilibrium atomistic description at finite temperature. This is accomplished by way of a variational mean field approximation for systematically averaging the dynamics of the atomistic system and by appending the resulting free-energy minimization problem to an energy-balance equation including an empirical heat conduction term. The main advantage of the method is that the max-ent probability distribution and the thermodynamic potentials are explicit and local in nature, which enables the modeling of non-equilibrium thermodynamic phenomena. Numerical validation tests reveal that the max-ent distribution, which can account for the anharmonic effects of the interaction potential, leads to a significant improvement in the predictions of the specific heat and the coefficient of thermal expansion over methods based on a quasi-harmonic approximation. The method is also capable of modeling behavior at high temperatures where the quasi-harmonic approximation fails.

The max-ent approximation scheme has been taken as a basis for developing a nonequilibrium finite temperature extension of the quasicontinuum method. The resulting scheme provides a means of seamlessly bridging the atomistic and continuum realms at finite temperature, including heat conduction. In the spirit of the quasicontinuum theory a full description of the behavior of the system is achieved once the interatomic potential and the heat conductivity are provided. A key property of the method is its ability to cap- 


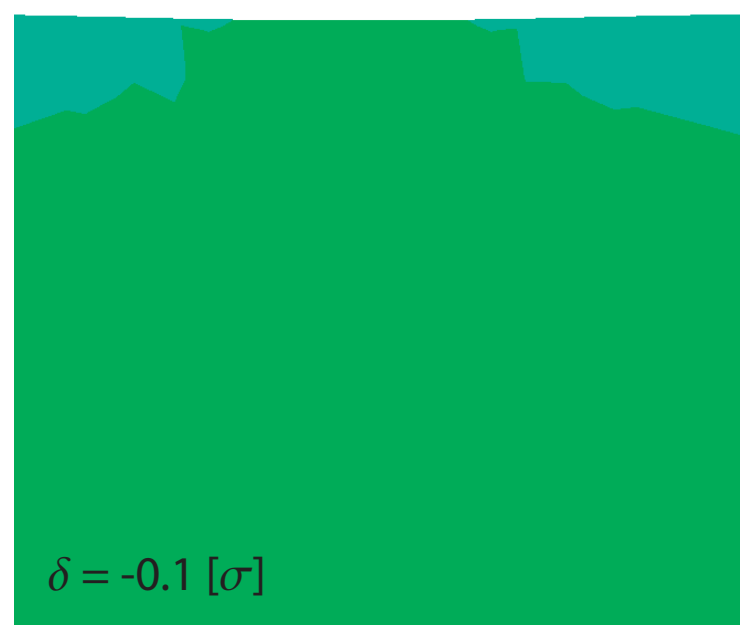

temperature
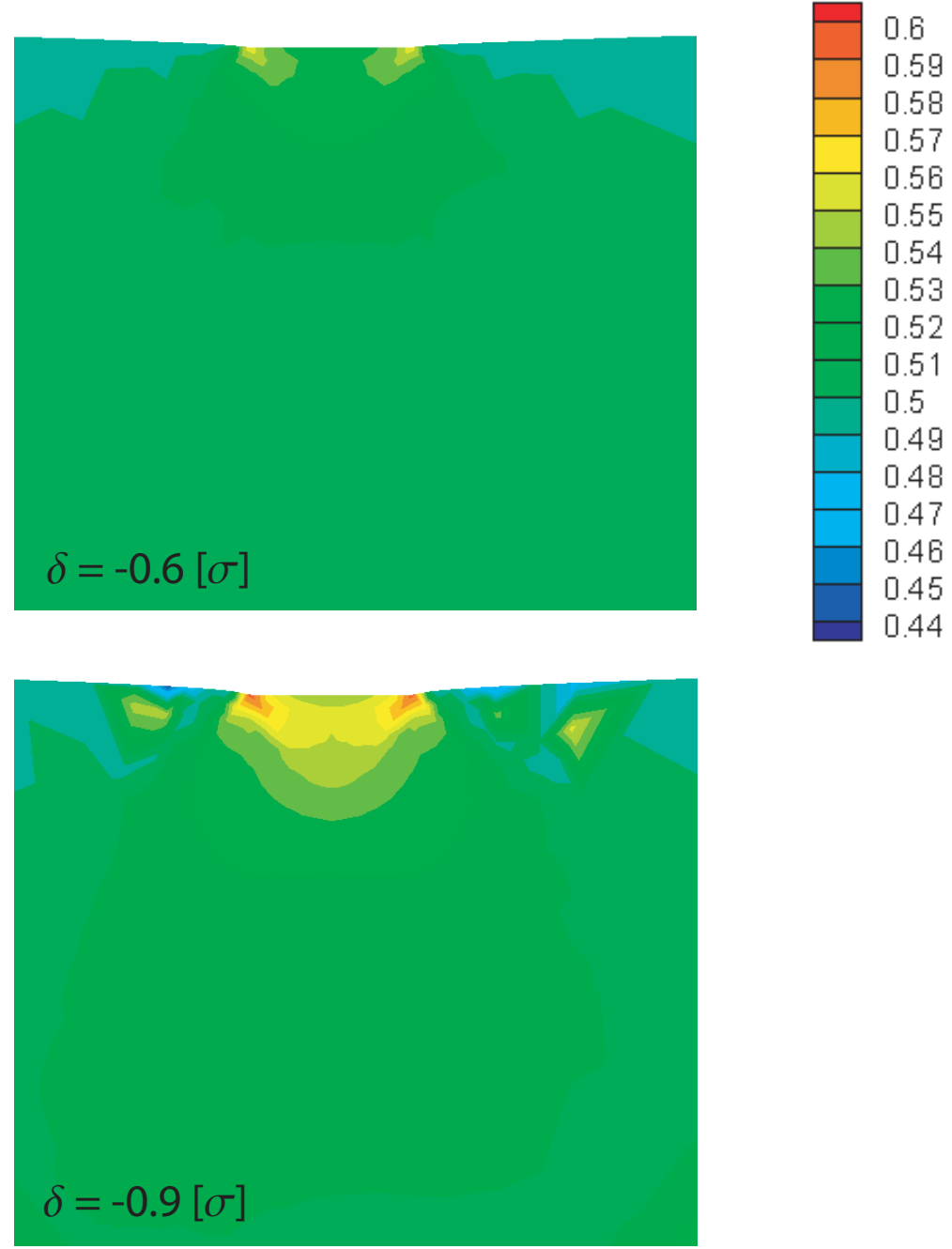

Figure 11.5: Snapshots showing the temperature profile of the cross-section A'B'C'D' under the indenter at different indentation depths during the simulation. The test is performed under adiabatic conditions. 


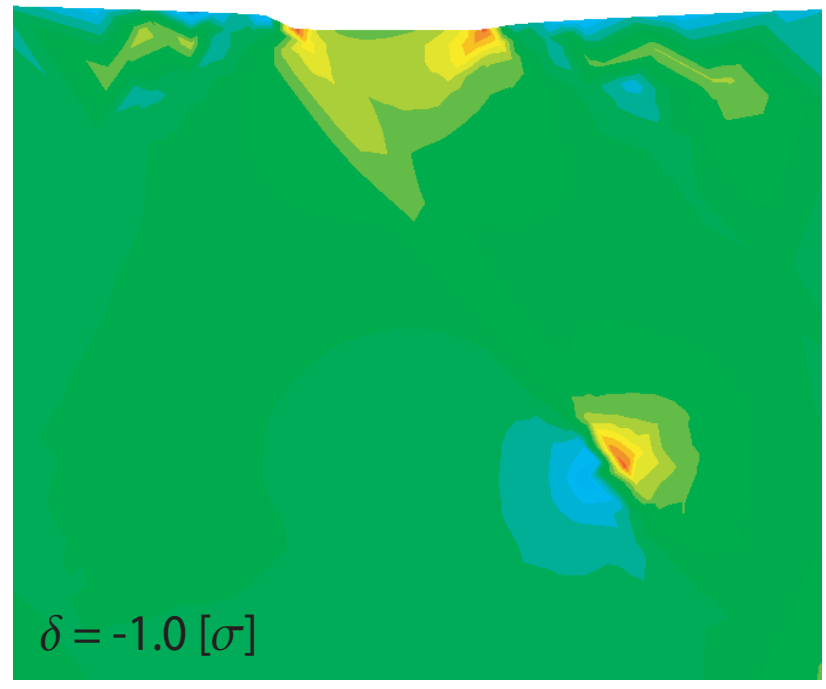

temperature
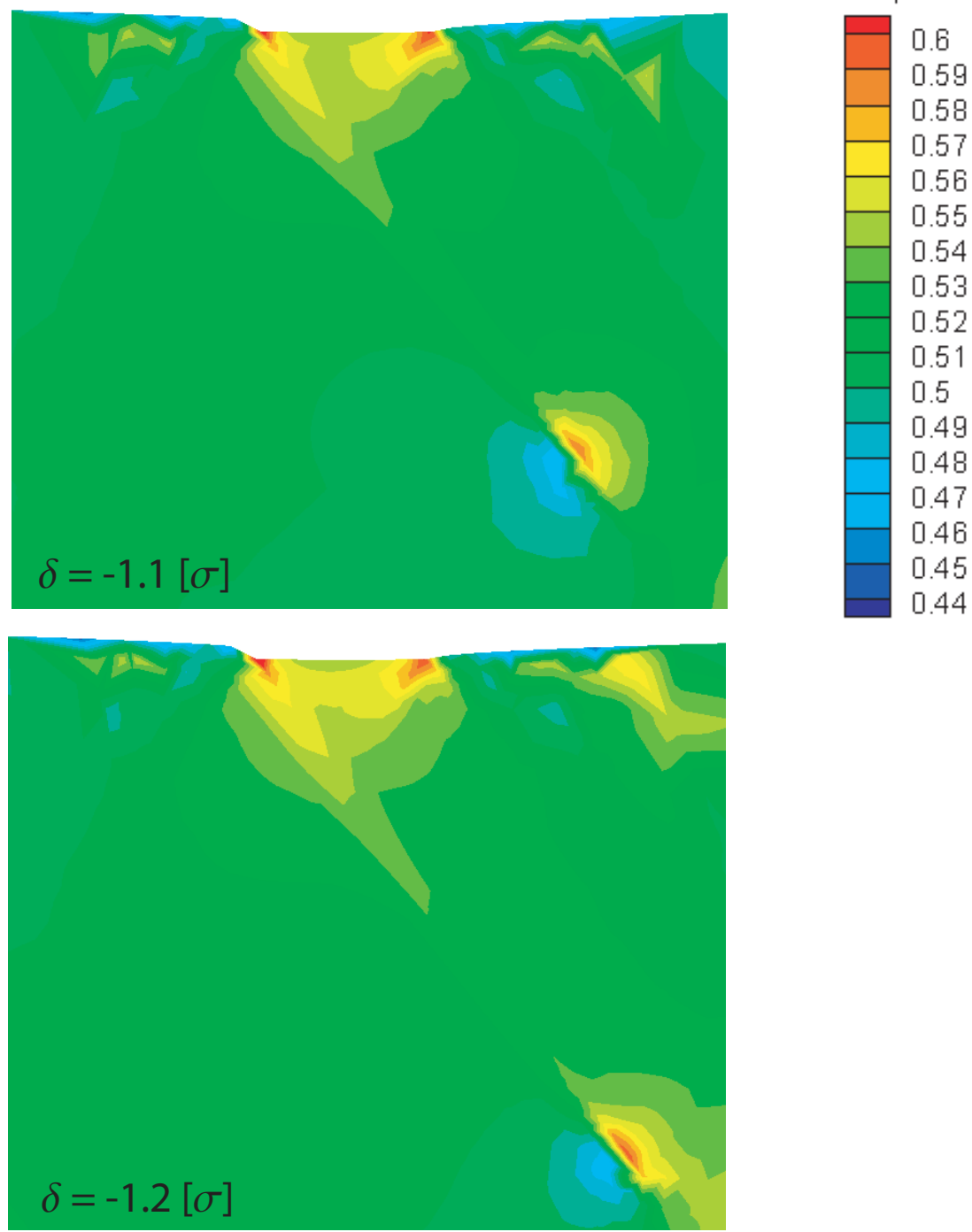

Figure 11.6: Snapshots at subsequent load increments showing the temperature of the cross-section A'B'C'D' profile under the indenter after a dislocation has nucleated. The test is performed under adiabatic conditions. 


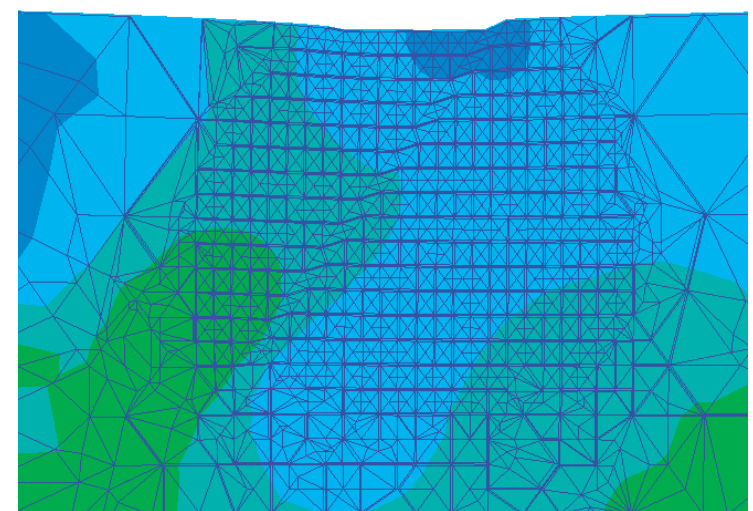

$\delta=-1.0[\sigma]$

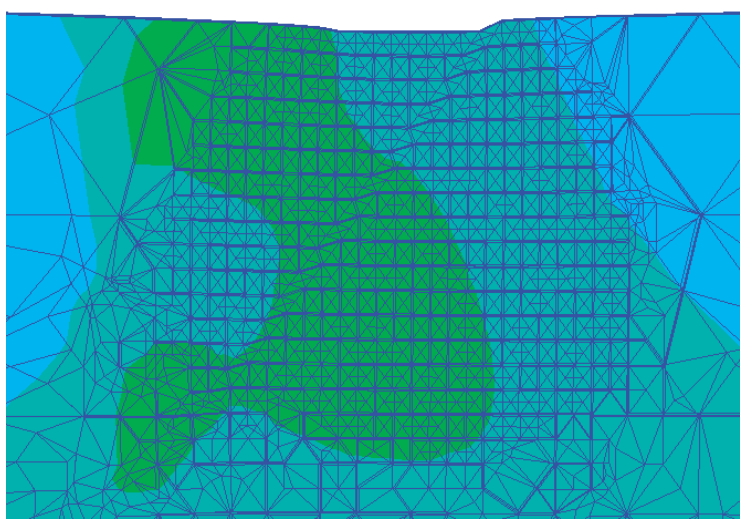

temperature

$\delta=-1.1[\sigma]$

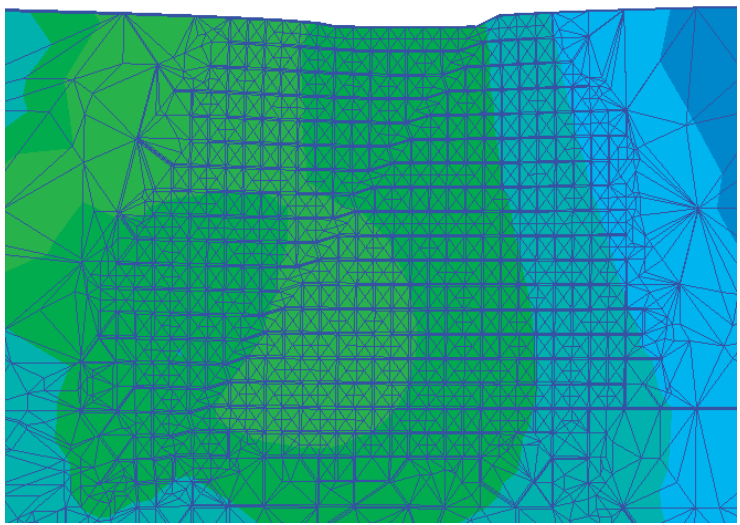

$$
\delta=-1.2[\sigma]
$$

Figure 11.7: Snapshots at subsequent load increments showing the temperature profile of the crosssection A'B'C'D' under the indenter after a dislocation has nucleated. The indentation velocity is $1.95 \mathrm{~m} / \mathrm{s}$. The negligible variation in temperature shows that the conditions are isothermal. 


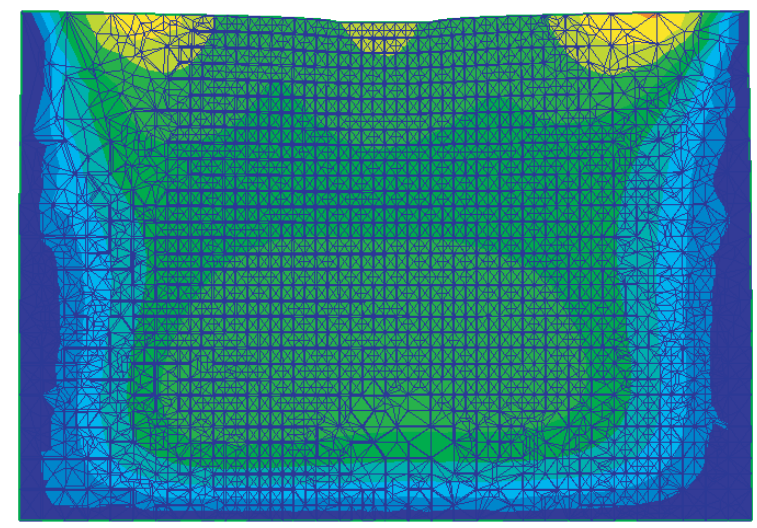

$\delta=-1.0[\sigma]$

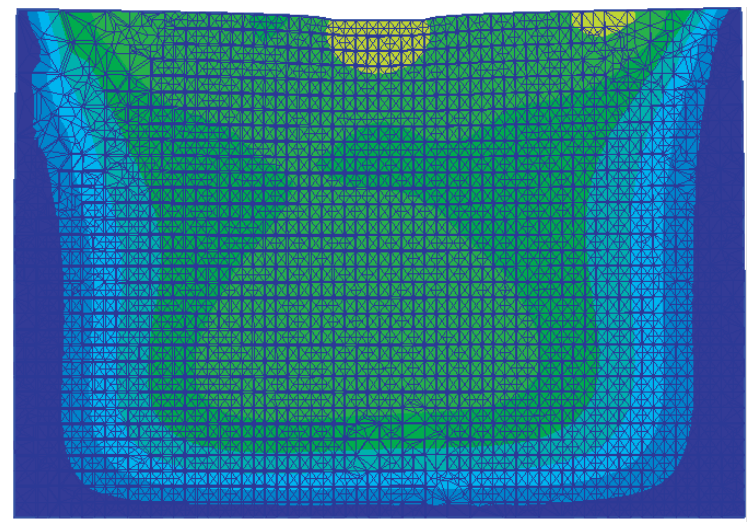

temperature

$\delta=-1.1[\sigma]$
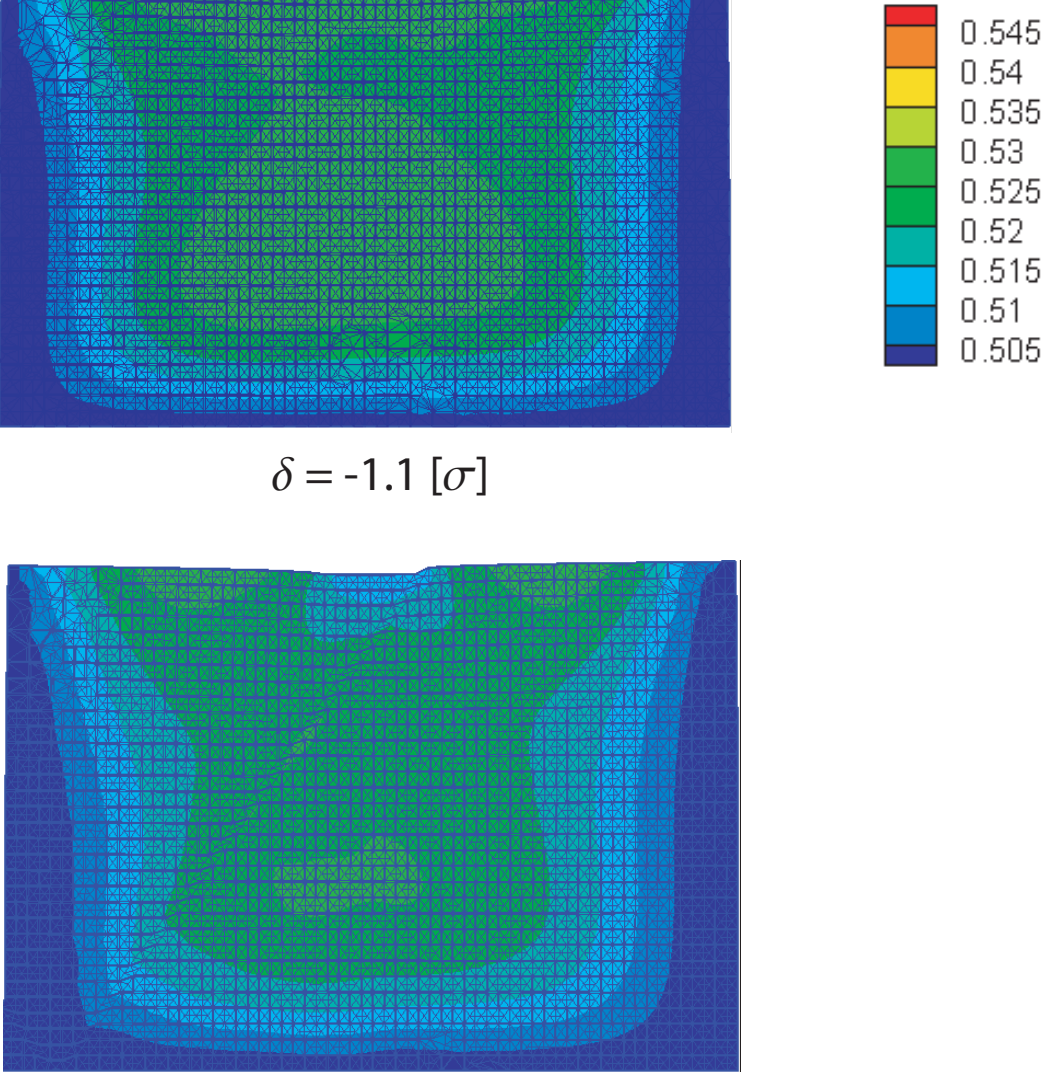

$$
\delta=-1.2[\sigma]
$$

Figure 11.8: Snapshots at subsequent load increments showing the temperature profile of the entire cross-section ABCD under the indenter just before and after a dislocation has nucleated. The indentation velocity is $19.5 \mathrm{~m} / \mathrm{s}$ which is close to the critical velocity. 

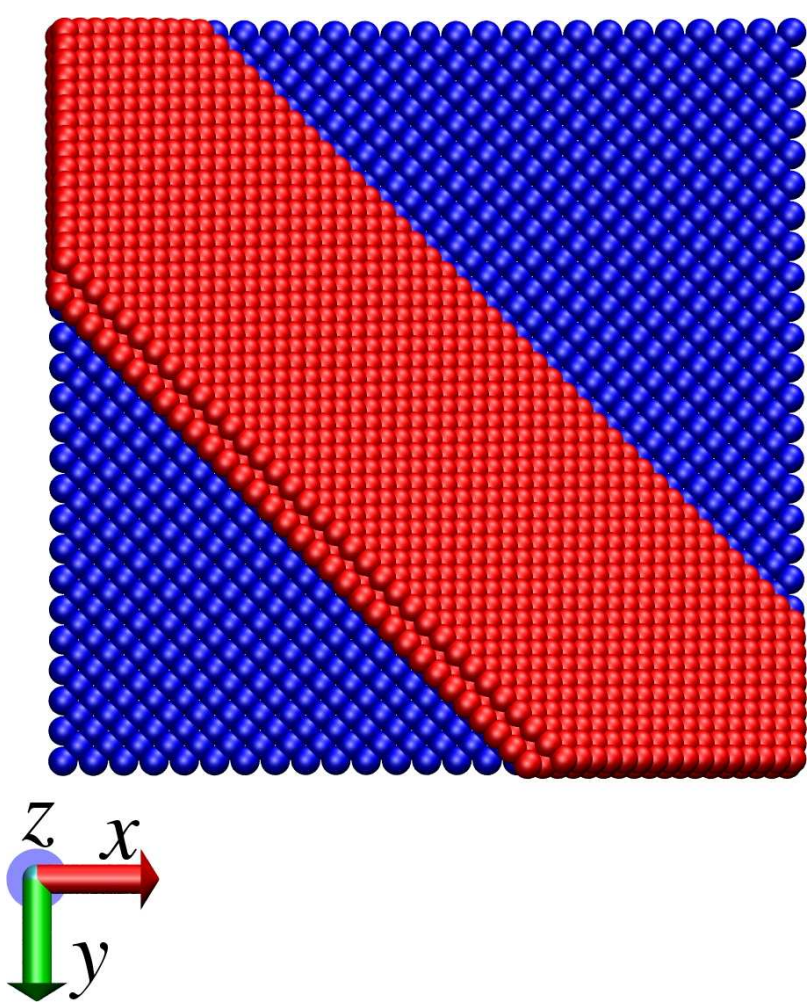

Figure 11.9: Dislocation structure under a rectangular indenter predicted by the quasicontinuum method at indentation depth $\delta=1.2[\sigma]$ under adiabatic conditions. The figure displays the energetic atoms (red) under the top surface of the crystal (blue).

ture microstructural evolution and the associated thermo-mechanically coupled behavior. In particular, the coupling of the free energy of the lattice to heat conduction has the effect of introducing an intrinsic time scale into the system. This intrinsic time scale in turn differentiates between slow and fast processes, with the response to former being predominantly isothermal and the response to the latter predominantly adiabatic. The nanoindentation tests presented in the foregoing exemplify this behavior. Thus, well below a well-defined characteristic indentation velocity dislocations are punched and move into the crystal in an ostensibly uniform temperature field, whereas well above the characteristic indentation velocity the dislocation cores carry along an autonomous temperature field resulting from local adiabatic heating. At intermediate indentation velocities a smooth transition is observed between these two extreme behaviors.

We close by pointing out possible directions for further extension of the approach. By allowing for arbitrary mean atomic momenta, the max-ent approach results in a temperaturedependent Hamiltonian governing the propagation of lattice phonons at finite temperature. However, there still remains a numerical issue of eliminating the spurious reflection of high frequency waves at the fine-coarse interface as they radiate into the coarse region and the 


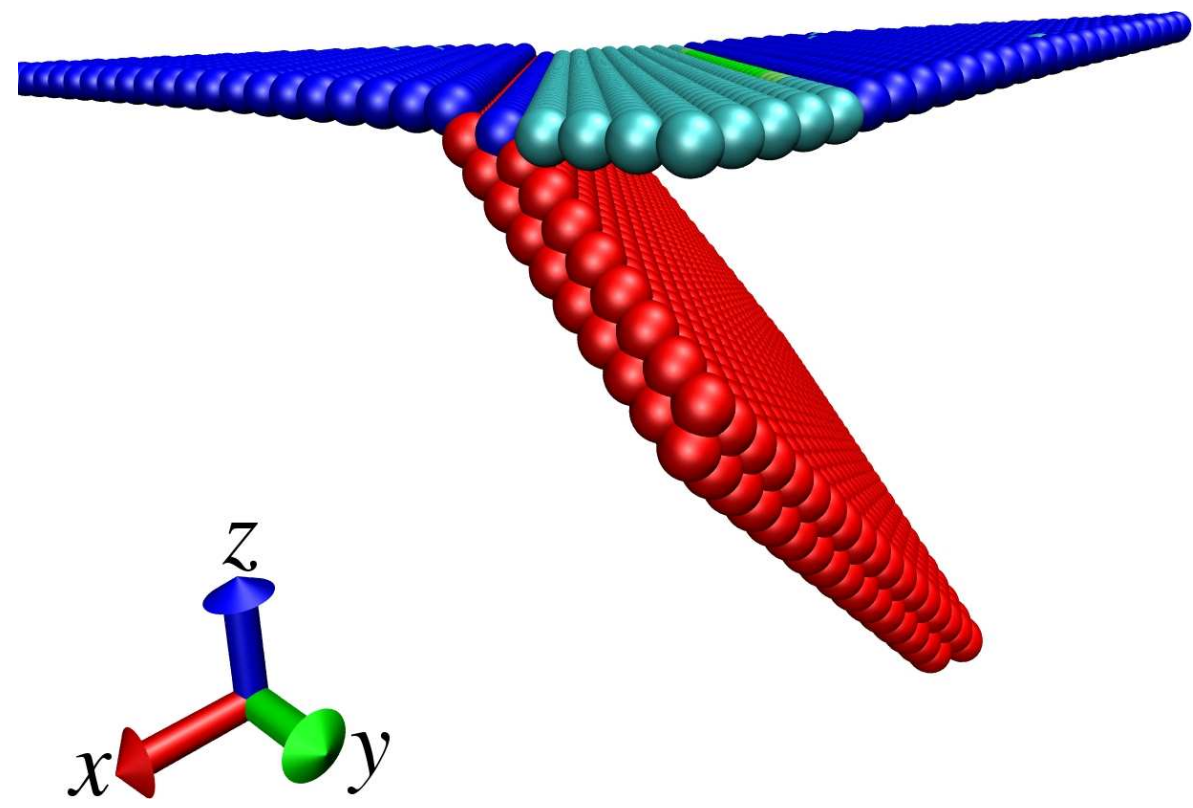

Figure 11.10: Another view of the dislocation structure in Figure (11.9) showing the stacking fault along the (111) plane. The figure displays the energetic atoms (red) under the top surface of the crystal (blue).

consequent non-physical heating of the atomistic region. Finally, we note that other transport phenomena, such as mass transport, should be amenable to a treatment similar to heat conduction. This extension would require the introduction of additional concentration and mass flux fields, the use of the max-ent formalism to determine concentration-dependent free energies and effective interatomic potentials, and the coupling of the resulting freeenergy minimization problem to a mass conservation equation including empirical diffusivity parameters.

\section{Acknowledgements}

We gratefully acknowledge the support of the Department of Energy through Caltech's ASCI ASAP Center for the Simulation of the Dynamic Response of Materials, the support received from the National Science Foundation through an ITR grant on Multiscale Modeling and Simulation and Caltech's Center for Integrative Multiscale Modeling and Simulation, and the support of the Army Research Laboratory through the CHSSI II Project on Scalable Quasi-Continuum Software for Advanced Mixed Atomistic-Continuum Simulation of Material Behavior at the Nanoscale. JK gratefully acknowledges the support of the U. S. Department of Energy through the Lawrence Livermore National Laboratory under Contract W-7405-Eng-48. 


\section{A Gauss quadrature for multiple integrals}

This appendix presents a brief summary of the third and fifth degree quadrature rules for multiple integrals in a space of dimension $n$ used in our calculations. The expressions and the quadrature tables were obtained from the work of A. H. Stroud [25]. In particular, we are interested in the following integrals with gaussian weighting functions:

$$
I(f)=\int_{-\infty}^{\infty} \cdots \int_{-\infty}^{\infty} f\left(x_{1}, \ldots, x_{n}\right) \exp \left[-x_{1}^{2}-\cdots-x_{n}^{2}\right] d x_{1} \ldots d x_{n} .
$$

An $M$-point numerical quadrature approximates the integral as

$$
I(f) \approx \sum_{k=1}^{M} f\left(\nu_{k}\right) W_{k},
$$

where $\nu_{k}$ is an $n$-dimensional vector at the $k^{\text {th }}$ quadrature point:

$$
\nu_{k}=\nu_{1 k}, \ldots, \nu_{n k}
$$

The expressions for the quadrature points and the associated weights are given below.

\section{A.1 Third degree quadrature}

This formula has $2 n$ points. The points and coefficients are obtained by requiring that the formula should integrate all monomials of degree $\leq 3$ exactly. Since the domain of integration is $\mathbb{R}^{n}$ and the gaussian weight has the property

$$
w(\boldsymbol{x})=w(-\boldsymbol{x}),
$$

the distribution of quadrature points is assumed to be fully symmetric. That is, we assume that the formula consists of $2 n$ points $\nu_{k}$ and $-\nu_{k}$ and that the coefficient of $\nu_{k}$ equals that of $-\nu_{k}$. Thus, for an $n$-dimensional space, the points are

$$
\begin{aligned}
& ( \pm r, 0, \ldots, 0) \\
& \vdots \\
& (0, \ldots, 0, \pm r)
\end{aligned}
$$

with the coefficient

$$
W_{k}=\frac{1}{2 n} V, \quad k=1, \ldots, n,
$$

where

$$
V=I(1)=\pi^{n / 2}, \quad r^{2}=\frac{n}{2} .
$$




\section{A.2 Fifth degree quadrature}

This formula has $n^{2}+n+2$ points. The formula is obtained by requiring that it be exact for all monomials of degree $\leq 5$. Owing to the symmetry of the domain, the formula consists of $\frac{1}{2}\left(n^{2}+n+2\right)$ points $\nu_{k}$ and their negatives, $-\nu_{k}$. The coefficient of $\nu_{k}$ equals that of $-\nu_{k}$. For an $n$-dimensional space, the $5^{\text {th }}$ degree quadrature points and their coefficients can be written as follows using 8 parameters:

$$
\begin{aligned}
& (\eta, \eta, \ldots, \eta, \eta) A \\
& (\lambda, \xi, \ldots, \xi, \xi) B \\
& \vdots \\
& (\xi, \xi, \ldots, \xi, \lambda) B \\
& (\mu, \mu, \gamma, \ldots, \gamma) C \\
& (\mu, \gamma, \mu, \ldots, \gamma) C \\
& \vdots \\
& (\gamma, \ldots, \gamma, \mu, \mu) C \\
& (-\eta,-\eta, \ldots,-\eta,-\eta) A \\
& (-\lambda,-\xi, \ldots,-\xi,-\xi) B \\
& \vdots \\
& (-\xi,-\xi, \ldots,-\xi,-\lambda) B \\
& (-\mu,-\mu,-\gamma, \ldots,-\gamma) C \\
& (-\mu,-\gamma,-\mu, \ldots,-\gamma) C \\
& \vdots \\
& (-\gamma, \ldots,-\gamma,-\mu,-\mu) C .
\end{aligned}
$$

The values of the eight parameters for different dimensions are provided in [24]. 


\section{References}

[1] T. Çağin, G. Dereli, M. Uludoğan, and M. Tomak. Thermal and mechanical properties of some fcc transition metals. Physical Review B, 59:3468-3473, 1999.

[2] P. M. Chaikin and T. C. Lubensky. Principles of condensed matter physics. Cambridge University Press, 1995.

[3] D. Chandler. Introduction to modern statistical mechanics. Oxford University Press, 1987.

[4] M. S. Daw and M. I. Baskes. Embedded-atom method: Derivation and application to impurities, surfaces and other defects in metals. Physical Review B, 29:6443-6453, 1984.

[5] S. R. deGroot and P. Mazur. Non-equilibrium thermodynamics. North-Holland, 1962.

[6] E. R. Dobbs and G. O. Jones. Theory and properties of solid argon. Rep. Prog. Phys., 20:516-564, 1957.

[7] L. M. Dupuy, E. B. Tadmor, R. E. Miller, and R. Phillips. Finite-temperature quasicontinuum: Molecular dynamics without all the atoms. Physical Review Letters, 95:0602021-060202-4, 2005.

[8] F. B. Hildebrand. Introduction to numerical analysis. Dover, 1987.

[9] T. J. R. Hughes. The finite element method: Linear static and dynamic finite element analysis. Dover, 2000.

[10] E. Jaynes. Information theory and statistical mechanics. Physical Review, 106:620-630, 1957.

[11] R. A. Johnson. Analytic nearest-neighbor model for fcc metals. Physical Review B, 37:3924-3931, 1988.

[12] D. Jou, J. Casas-Vázquez, and G. Lebon. Extended irreversible thermodynamics. Springer, 1996.

[13] C. L. Kelchner, S. J. Plimpton, and J. C. Hamilton. Dislocation nucleation and defect structure during surface nanoindentation. Physical Review B, 58:11085-11088, 1998.

[14] J. Knap and M. Ortiz. An analysis of the quasicontinuum method. Journal of the Mechanics and Physics of Solids, 49:1899-1923, 2001.

[15] Y. Kulkarni. Coarse-graining of atomistic description at finite temperature. PhD thesis, California Institute of Technology, October 2006.

[16] C. Lanczos. The variational principles of mechanics. Dover, 1986. 
[17] L. D. Landau and E. M. Lifshitz. Statistical Physics: Part 1. Elsevier ButterworthHeinemann, 3rd edition, 1980.

[18] R. LeSar, R. Najafabadi, and D. Srolovitz. Finite-temperature defect properties from free-energy minimization. Physical Review Letters, 63:624-627, 1989.

[19] R. E. Miller and E. B. Tadmor. The quasicontinuum method: Overview, applications and current directions. Journal of Computer-Aided Materials Design, 9:203-239, 2002.

[20] F. C. Nix and D. MacNair. The thermal expansion of pure metals: Copper, gold, aluminum, nickel and iron. Physical Review, 60:597-605, 1941.

[21] O. G. Peterson, D. N. Batchelder, and R. O. Simmons. Measurements of x-ray lattice constant, thermal expansivity, and isothermal compressibility of argon crystals. Physical Review, 150:703-711, 1966.

[22] V. Shenoy, V. Shenoy, and R. Phillips. Finite temperature quasicontinuum methods. Materials Research Society Symposium Proceedings, 538:465-471, 1999.

[23] V. B. Shenoy, R. Phillips, and E. B. Tadmor. Nucleation of dislocations beneath a plane strain indenter. Journal of the Mechanics and Physics of Solids, 48:649-673, 2000.

[24] A. H. Stroud. Some fifth degree integration formulas for symmetric regions ii. $\mathrm{Nu}$ merische Mathematik, 9:460-468, 1967.

[25] A. H. Stroud. Approximate Calculation of Multiple Integrals. PrenticeHall, 1971.

[26] A. P. Sutton and J. Chen. Long range finnis-sinclair potentials. Philosophical Magazine Letters, 61:139-146, 1990.

[27] E. B. Tadmor, M. Ortiz, and R. Phillips. Quasicontinuum analysis of defects in solids. Philosophical Magazine, 73:1529-1563, 1996.

[28] J. H. Weiner. Statistical mechanics of elasticity. Dover, 2002.

[29] E. Y. Wu. A method for treating thermal expansion effects in molecular dynamics simulation for solids. Journal of Physics: Condensed Matter, 2:9335-9344, 1990.

[30] Q. Yang, L. Stainier, and M. Ortiz. A variational formulation of the coupled thermomechanical boundary-value problem for general dissipative solids. Journal of the $\mathrm{Me}$ chanics and Physics of Solids, 54:401-424, 2006.

[31] D. N. Zubarev. Non-equilibrium statistical thermodynamics. Consultants Bureau, 1974. 